\title{
Holographic charm and bottom pentaquarks. I. Mass spectra with spin effects
}

\author{
Yizhuang Liu๑* \\ Institute of Theoretical Physics, Jagiellonian University, 30-348 Kraków, Poland \\ Maciej A. Nowak ${ }^{\dagger}$ \\ Institute of Theoretical Physics and Mark Kac Center for Complex Systems Research, Jagiellonian University, \\ 30-348 Kraków, Poland \\ Ismail Zahed \\ Center for Nuclear Theory, Department of Physics and Astronomy, Stony Brook University, \\ Stony Brook, New York 11794-3800, USA
}

(Received 7 September 2021; accepted 31 October 2021; published 17 December 2021)

\begin{abstract}
We revisit the three nonstrange pentaquarks $\left[\frac{1}{2} \frac{1}{2}^{-}\right]_{S=0,1}$ and $\left[\frac{1}{2}^{-}\right]_{S=1}$ predicted using the holographic dual description, where chiral and heavy quark symmetry are manifest in the triple limit of a large number of colors, large quark mass and strong 't Hooft gauge coupling. In the heavy quark limit, the pentaquarks with internal heavy quark spin $S$ are all degenerate. The holographic pentaquarks are dual to an instanton bound to heavy mesons in bulk, without the shortcomings related to the nature of the interaction and the choice of the hard core inherent to the molecular constructions. We explicitly derive the spin-spin and spin-orbit couplings arising from next to leading order in the heavy quark mass, and lift totally the internal spin degeneracy, in fair agreement with the newly reported charmed pentaquarks from LHCb. New charm and bottom pentaquark states are predicted.
\end{abstract}

DOI: 10.1103/PhysRevD.104.114021

\section{INTRODUCTION}

Recently the LHCb Collaboration revisited its analysis of the pentaquark states using a ninefold increase in reconstructed $\Lambda_{b}^{0} \rightarrow J / \Psi p K^{-}$decays from the LHCb Run-2 data batch at $13 \mathrm{TeV}$ [1]. The LHCb new high statistics analysis shows that the previously reported $P_{c}^{+}$(4450) [2] splits into two narrow peaks $P_{c}^{+}(4440)$ and $P_{c}^{+}(4457)$ below the $\Sigma_{c}^{+} \bar{D}^{* 0}$ threshold, with the appearance of a new and narrow $P_{c}^{+}$(4312) state below the $\Sigma_{c}^{+} \bar{D}^{0}$. The evidence for the previously reported state $P_{c}^{+}$(4380) [2] has weakened.

We regard the new LHCb data as evidence that supports the three lowest nonstrange pentaquarks with spin-isospin assignments $\left[\frac{1}{2} \frac{1}{2}^{-}\right]_{S=0,1}$ and $\left[\frac{1}{2} \frac{3}{2}\right]_{S=1}$ predicted by holography [3], in the triple limit of a large number of colors, strong 't Hooft gauge coupling and a large quark mass. More importantly, we will show below that the degeneracy in the

\footnotetext{
*yizhuang.liu@uj.edu.pl

maciej.a.nowak@uj.edu.pl

*ismail.zahed@stonybrook.edu
}

Published by the American Physical Society under the terms of the Creative Commons Attribution 4.0 International license. Further distribution of this work must maintain attribution to the author(s) and the published article's title, journal citation, and DOI. Funded by SCOAP ${ }^{3}$. internal heavy quark spin $S=1$ is lifted by spin-orbit effects at next to leading order in the heavy quark mass as heavy quark symmetry is broken, in fair agreement with the new data. Furthermore, we regard the closeness of the pentaquarks $P_{c}^{+}(4457)$ and $P_{c}^{+}(4312)$ to the $\Sigma_{c}^{+} \bar{D}^{* 0}$ and $\Sigma_{c}^{+} \bar{D}^{0}$ thresholds respectively, as further evidence in support of this construction, as both thresholds coalesce in the heavy quark limit.

Pentaquark states with hidden charm were initially suggested in [4,5], and since have been addressed by many (see [6-12] and references therein). In short, the current descriptions range from pentaquarks made of compact diquarks $[13,14]$, to hadrocharmonia [15] and loosely bound hadronic molecules [16] (see also references therein). Heavy pentaquarks, as multiquark states composed of heavy and light quarks, fall outside the realm of the canonical quark model. Their description calls for a novel hadronic paradigm with manifest chiral and heavy quark symmetry.

It is well established that chiral symmetry dictates most of the interactions between light quarks, while heavy quark symmetry organizes the spin interactions between heavy quarks $[17,18]$. Both symmetries are intertwined by the phenomenon of chiral doubling [19-21], as shown experimentally in $[22,23]$. Therefore, a theoretical approach to the multiquark states should have manifest chiral and heavy 
quark symmetry, a clear organizational principle in the confining regime, and should address concisely the multibody bound state problem.

The holographic principle in general [24,25], and the D4-D8-D $\overline{8}$ holographic setup in particular [26] provide a framework for addressing QCD in the infrared in the double limit of a large number of colors and strong 't Hooft gauge coupling $\lambda=g_{\mathrm{YM}}^{2} N_{c}$. It is confining and exhibits spontaneous chiral symmetry breaking geometrically. The light meson sector is well described by an effective action with manifest chiral symmetry and very few parameters, yet totally in line with more elaborate effective theories of QCD [27]. The same setup can be minimally modified to account for the description of heavy-light mesons, with manifest heavy quark symmetry [3,28-31].

Light and heavy-light baryons are dual to instantons and instanton-heavy meson bound states in bulk [32-37], providing a robust geometrical approach to the multibody bound state problem. The holographic construction provides a dual realization of the chiral soliton approach and its bound state variants $[38,39]$, without the shortcomings of the derivative expansion. It is a geometrical realization of the molecular approach [4,5], without the ambiguities of the nature of the meson exchanges, and the arbitrariness in the choice of the many couplings and form factors [40]. Alternative holographic models for the description of heavy hadrons have been developed in [41,42].

The organization of the paper is as follows: In Sec. II we recall the essential aspects of the $N_{f}=2$ heavy-light effective action in leading order in the heavy quark mass introduced in [3,28]. In Sec. III we extend this analysis at next to leading order in the heavy quark mass for the bound heavy baryons seeded by instantons in bulk. In Sec. IV we detail the spin-orbit and spin-spin effects for the heavy baryons and their exotics. The induced quantum effective potentials are made explicit in Sec. V. In Sec. VI we derive the holographic mass formula for the heavy-light baryons and their exotic pentaquarks, including the spin contributions. By adjusting the chief Kaluza-Klein scale used in $[3,29]$, a more refined heavy-baryon spectrum emerges, including the newly reported charmed pentaquarks by LHCb. Our conclusions are in Sec. VII. A number of Appendixes are added to support the various results.

\section{HOLOGRAPHIC HEAVY-LIGHT EFFECTIVE ACTION}

The D4-D8-D $\overline{8}$ setup for light flavor branes is standard [26]. The minimal modification that accommodates heavy mesons makes use of an extra-heavy brane, as discussed in [3,28,29]. It consists of $N_{f}$ light D8-D $\overline{8}$ branes (L) and one heavy $(\mathrm{H})$ probe brane in the cigar-shaped geometry that spontaneously breaks chiral symmetry. We assume that the L-brane world volume consists of $R^{4} \times S^{1} \times S^{4}$ with $[0-9]$ dimensions. The light 8 -branes are embedded in the $[0-3+5-9]$ dimensions and set at the antipodes of $S^{1}$, which lies in the fourth dimension. The warped [5-9]-space is characterized by a finite size $R$ and a horizon at $U_{K K}$.

\section{A. Dirac-Born-Infeld (DBI) action}

The effective action on the probe L-branes consists of the non-Abelian DBI and Chern-Simons action. After integrating over the $S^{4}$, the leading contribution in $1 / \lambda$ to the DBI action is

$S_{\mathrm{DBI}} \approx-\kappa \int d^{4} x d z \operatorname{Tr}\left(\mathbf{f}(z) \mathbf{F}_{\mu \nu} \mathbf{F}^{\mu \nu}+\mathbf{g}(z) \mathbf{F}_{\mu z} \mathbf{F}^{\nu z}\right)$.

The warping factors are

$$
\mathbf{f}(z)=\frac{R^{3}}{4 U_{z}}, \quad \mathbf{g}(z)=\frac{9}{8} \frac{U_{z}^{3}}{U_{K K}},
$$

with $U_{z}^{3}=U_{K K}^{3}+U_{K K} z^{2}$, and $\kappa \equiv a \lambda N_{c}$ and $a=1 /$ $\left(216 \pi^{3}\right)$ [26]. All dimensions are in units of $M_{K K}$ (Kaluza-Klein scale) unless given explicitly. Our conventions are $(-1,1,1,1,1)$ with $A_{M}^{\dagger}=-A_{M}$ and the labels $M, N$ running over $\mu, z$ only in this section. The effective fields in the field strengths are $[3,28]$

$\mathbf{F}_{M N}=\left(\begin{array}{cc}F_{M N}-\Phi_{[M} \Phi_{N]}^{\dagger} & \partial_{[M} \Phi_{N]}+A_{[M} \Phi_{N]} \\ -\partial_{[M} \Phi_{N]}^{\dagger}-\Phi_{[M}^{\dagger} A_{N]} & -\Phi_{[M}^{\dagger} \Phi_{N]}\end{array}\right)$.

The matrix valued 1-form gauge field is

$$
\mathbf{A}=\left(\begin{array}{cc}
A & \Phi \\
-\Phi^{\dagger} & 0
\end{array}\right)
$$

For $N_{f}=2$, the naive Chern-Simons 5-form is

$$
S_{\mathrm{CS}}=\frac{i N_{c}}{24 \pi^{2}} \int_{M_{5}} \operatorname{Tr}\left(A F^{2}-\frac{1}{2} A^{3} F+\frac{1}{10} A^{5}\right) .
$$

We note that only for $N_{f}>2$ does it fail to reproduce the correct transformation law under the combined gauge and chiral transformations [35]. In particular, when addressing the $N_{f}=3$ baryon spectra, Eq. (5) does not reproduce the important hypercharge constraint [35], but can be minimally modified to do that.

For $N_{f}$ coincidental branes, the $\Phi$ multiplet is massless, but for separated branes they are massive with the additional contribution

$$
\frac{1}{2} m_{H}^{2} \operatorname{Tr}\left(\Phi_{M}^{\dagger} \Phi_{M}\right)
$$

The value of $m_{H}$ is related to the separation between the light and heavy branes, which is about the length of the HL string. 
It is related to the heavy meson masses $M_{D}=1870 \mathrm{MeV}$ (charmed) and $M_{B}=5279 \mathrm{MeV}$ (bottomed) through [28]

$$
M_{D, B}=m_{H}+\frac{M_{K K}}{2 \sqrt{2}} .
$$

Given $M_{K K}$ and $M_{D, B}$, the mass parameter $m_{H}$ is therefore totally fixed.

While the use of one heavy brane is well suited for single heavy baryons, doubly heavy baryons require a priori two heavy branes. However, in the heavy quark limit and to order $1 / m_{H}$, including the spin-orbit effects as we will discuss below, there is no need to double the heavy branes. Such doubling will be needed if e.g., spin-spin interactions are retained to order $1 / m_{H}^{2}$.

\section{B. Light fields}

In the coincidental brane limit, light baryons are interchangeably described as a flavor instanton or a D4 brane wrapping the $S^{4}$. The instanton mass is $M_{0}=8 \pi^{2} \kappa$ in units of $M_{K K}$. The instanton size is small with $\rho \sim 1 / \sqrt{\lambda}$ after balancing the order $\lambda$ bulk gravitational attraction with the subleading and of order $\lambda^{0} \mathrm{U}(1)$ induced topological repulsion [26]. The bulk instanton is described by the $\mathrm{O}$ (4) gauge field

$$
A_{M}(y)=-\bar{\sigma}_{M N} \partial_{N} F(y),\left.\quad F_{z m}(y)\right|_{|y|=R}=0 .
$$

From here on $M, N$ run only over $1,2,3, z$ unless specified otherwise. If $\rho \sim 1 / \sqrt{\lambda}$ is the typical size of these tunneling configurations, then it is natural to recast the DBI action using the rescaling

$$
\begin{aligned}
\left(x_{0}, x_{M}\right) & \rightarrow\left(x_{0}, x_{M} / \sqrt{\lambda}\right), \sqrt{\lambda} \rho \rightarrow \rho,\left(A_{0}, A_{M}\right) \\
& \rightarrow\left(A_{0}, \sqrt{\lambda} A_{M}\right) .
\end{aligned}
$$

The rescaled fields satisfy the equations of motion

$$
D_{M} F_{M N}=0, \quad \partial_{M}^{2} A_{0}=-\frac{1}{32 \pi^{2} a} F_{a M N} \star F_{a M N},
$$

with the use of the Hodge dual notation.

\section{Heavy-light fields}

Let $\left(\Phi_{0}, \Phi_{M}\right)$ be the pair of heavy quantum fields that bind to the tunneling configuration above. If again $\rho \sim 1 / \sqrt{\lambda}$ is their typical size, then it is natural to recast the heavy-light part of the DBI action using the additional rescaling

$$
\left(\Phi_{0}, \Phi_{M}\right) \rightarrow\left(\Phi_{0}, \sqrt{\lambda} \Phi_{M}\right) .
$$

The interactions between the light gauge fields $\left(A_{0}, A_{M}\right)$ and the heavy fields $\left(\Phi_{0}, \Phi_{M}\right)$ to quadratic order split to several contributions $[3,28]$

$$
\mathcal{L}=a N_{c} \lambda \mathcal{L}_{0}+a N_{c} \mathcal{L}_{1}+\mathcal{L}_{\mathrm{CS}}
$$

which are quoted in Eq. (A1). We start by recalling the leading contributions in $1 / m_{H}$ stemming from Eq. (12), as thoroughly discussed in [3,29]. For that, we split $\Phi_{M}=\phi_{M} e^{-i m_{H} x_{0}}$ for particles $\left(m_{H} \rightarrow-m_{H}\right.$ for antiparticles). The leading-order contribution takes the form

$\mathcal{L}_{0}=-\frac{1}{2}\left|f_{M N}-\star f_{M N}\right|^{2}+2 \phi_{M}^{\dagger}\left(F_{M N}-\star F_{M N}\right) \phi_{N}$,

subject to the constraint equation $D_{M} \phi_{M}=0$ with

$$
f_{M N}=\partial_{[M} \phi_{N]}+A_{[M} \phi_{N]},
$$

while the subleading contributions in Eq. (12) to order $\lambda^{0} m_{H}$ simplify to

$$
\frac{\mathcal{L}_{1}}{a N_{c}} \rightarrow 4 m_{H} \phi_{M}^{\dagger} i D_{0} \phi_{M}, \quad \mathcal{L}_{\mathrm{CS}} \rightarrow \frac{m_{H} N_{c}}{16 \pi^{2}} \phi_{M}^{\dagger} \star F_{M N} \phi_{N} .
$$

For self-dual light gauge fields with $F_{M N}=\star F_{M N}$, the last contribution in Eq. (13) vanishes, and the minimum is reached for $f_{M N}=\star f_{M N}$. This observation when combined with the transversality condition for $D_{M} \phi_{M}=0$, amounts to a first-order equation for the combination $\psi=\bar{\sigma}_{M} \phi_{M}$ with $\sigma_{M}=(i, \vec{\sigma})$, i.e.,

$$
\sigma_{M} D_{M} \psi=D \psi=0
$$

as noted in $[3,28]$. In a self-dual gauge configuration, the heavy spin-1 meson transmutes to a massless spin- $\frac{1}{2}$ spinor that is Bogomol'nyi-Prasad-Sommerfeld bound in leading order.

\section{THE ORDER $1 / m_{H}^{2}$ LAGRANGIAN}

To account for the spin effects and the breaking of heavy quark symmetry we need to account for the $1 / m_{H}$ contributions to Eqs. (12)-(15). This will be sought by restricting the quantum and heavy fields to the quantum moduli. More specifically, we choose to parametrize the fields using

$$
\begin{aligned}
A_{M}(t, x) & =V\left(A_{M}^{c l}-i \partial_{M}\right) V^{-1}, \quad A_{0}(t, x)=0 \\
\Phi_{M}(t, x) & =\frac{e^{-i m_{H} t}}{\sqrt{16 m_{H} a N_{c}}} V(t, x) f(X(t), Z(t)) \bar{\sigma}_{M} \chi(t),
\end{aligned}
$$

which is equivalent to 
$A_{M}(t, x)=A_{M}^{\mathrm{cl}}(X(t), Z(t)), \quad A_{0}(t, x)=-i V \partial_{t} V^{-1} \equiv \Phi$,

$\Phi_{M}(t, x)=f(X(t), Z(t)) \bar{\sigma}_{M} \chi(t)$

after gauge transformation. The $\Phi$ is parametrized as

$$
\Phi=-\dot{X}_{N} A_{N}^{\mathrm{cl}}+\chi^{a} \Phi_{a}
$$

where $\Phi_{a}$ diagonalizes $D_{M}^{\mathrm{cl}} D_{M}^{\mathrm{cl}} \Phi_{a}$ and where

$$
\chi^{a}=\operatorname{tr}\left(\tau^{a} \mathbf{a}^{-1} \mathbf{a}\right)
$$

are expressed in terms of the collective variables $\mathbf{a} \in \mathrm{SU}(2)$ for a rigid $S U(2)$ rotation. The temporal component $\Phi_{0}$ satisfies the constraint

$$
\begin{aligned}
& \left(-D_{M}^{2}+m_{H}^{2}\right) \Phi_{0}+2 F_{M 0} \Phi_{M}-\frac{i}{16 \pi^{2} a} F_{P Q}\left(\partial_{P}+A_{P}\right) \Phi_{Q} \\
& =0
\end{aligned}
$$

and in leading order in $1 / m_{H}$ can be ignored.

Inserting the expansion (18) into Eq. (A1) yields the quadratic $1 / m_{H}$ contributions,

$$
\mathcal{L}_{\text {quadratic }}=\frac{1}{8 m_{H}} \chi^{\dagger} \sigma_{M} f\left(\partial_{t}-A_{0}-\Phi\right)\left(\partial_{t}-A_{0}-\Phi\right) \bar{\sigma}_{M} f \chi-\tilde{\mathcal{L}}_{1}+\tilde{\mathcal{L}}_{\mathrm{CS}}
$$

Here $\tilde{\mathcal{L}}_{\mathrm{CS}}$ contains only $\Phi_{M}$. Each of the contributions in Eq. (22) is discussed in Appendix A. Combining the results of Eqs. (A21), (A50), and (A56) we have for the quadratic contributions to order $\frac{1}{m_{H}}$

$$
\begin{aligned}
\mathcal{L}_{\text {quadratic }}= & \frac{1}{m_{H}}\left(\frac{c_{1}}{\pi^{4} a^{2} \rho^{4}} \chi^{\dagger} \chi+i \frac{c_{2}}{\pi^{2} a \rho^{2}} \chi^{\dagger} \dot{\chi}+i \frac{c_{3}}{\pi^{2} a \rho^{2}} \chi^{\dagger} \tau^{a} \chi \chi^{a}+\frac{\dot{\chi}^{\dagger} \dot{\chi}}{2}\right) \\
& -\frac{37+12 \frac{Z^{2}}{\rho^{2}}}{192 m_{H}} \chi^{\dagger} \chi+\left(\frac{1}{4 m_{H}} \frac{\dot{\rho}^{2}}{\rho^{2}}+\frac{\dot{a}_{I}^{2}}{4 m_{H}}+\frac{\dot{X}^{2}}{4 m_{H} \rho^{2}}\right) \chi^{\dagger} \chi,
\end{aligned}
$$

with the constants fixed to

$$
\begin{aligned}
& c_{1}=\frac{13}{3840}+\frac{7}{1280}=\frac{17}{1920}, \\
& c_{2}=\frac{2}{32} \\
& c_{3}=\frac{1}{128}+\frac{1}{80}=\frac{13}{640} .
\end{aligned}
$$

In the meantime, one has also to take into account the Chern-Simons term contribution

$$
-\frac{i}{16 \pi^{2}} \epsilon_{M N P Q} \Phi_{M}^{\dagger} \Phi_{N} \Phi_{P}^{\dagger} \partial_{t} \Phi_{Q}+\text { c.c. }
$$

which is

$$
\mathcal{L}_{\text {quartic,CS }}=-\frac{N_{c}}{5 m_{H} m_{y}^{2} \rho^{4}} \chi^{\dagger} \tau^{a} \chi \chi^{\dagger} \tau^{a} \chi
$$

where $m_{y}=16 \pi^{2} a$.

The above analysis ignores the Coulomb backreaction (repulsion from the bound charged fields) as we discussed in $[3,28]$ and can lead to instabilities. In Appendix B we detail the backreaction from the Coulomb field with the final result for Eq. (23) to order $\mathcal{O}\left(1 / m_{H}^{2}\right)$,

$$
\begin{aligned}
\mathcal{L} \rightarrow & i \chi^{\dagger} \dot{\chi}+\frac{1}{2 m_{H}} \dot{\chi}^{\dagger} \dot{\chi}+\frac{78}{5 m_{H} \tilde{\rho}^{2}} i \chi^{\dagger} \tau^{a} \chi \chi^{a}-\frac{12}{5 m_{H} \tilde{\rho}^{4}} \vec{S}^{2} \\
& -\frac{37+12 \frac{Z^{2}}{\rho^{2}}}{192 m_{H}} n+\frac{1}{\tilde{\rho}^{2}}\left(-\frac{18}{5}+\frac{9}{2} n-\frac{2}{3} n^{2}\right) \\
& +\frac{1}{m_{H} \tilde{\rho}^{4}}\left(\frac{102}{5} n-\frac{56}{5} n^{2}+\frac{4}{3} n^{3}+j \tilde{\rho}^{2}\left(\frac{9}{2}-\frac{4 n}{3}\right)\right) \\
& +\frac{1}{m_{H}^{2} \tilde{\rho}^{6}}\left(-\frac{128 n^{4}}{45}+\frac{376 n^{3}}{15}-\frac{4017 n^{2}}{70}\right. \\
& \left.-j n \tilde{\rho}^{2}\left(\frac{56}{5}-\frac{8}{3} n\right)-\frac{2}{3} j^{2} \tilde{\rho}^{4}\right),
\end{aligned}
$$

with

$$
j=\frac{i}{2}\left(\chi^{\dagger} \dot{\chi}-\dot{\chi}^{\dagger} \chi\right), \quad n=\chi^{\dagger} \chi .
$$

This is the first major result of this paper. We now study the quantization of Eq. (27) and the ensuing heavy-light baryonic spectra.

\section{QUANTUM SPIN EFFECTS}

\section{A. Spin-orbit effect}

The first major spin contribution occurs through the spinangular momentum coupling $\chi^{a} \chi^{\dagger} \tau^{a} \chi$. Recall that $\chi^{a}$ in modular variables is 


$$
\chi^{a}=2 i\left(a_{4} \dot{a}_{a}-\dot{a}_{4} a_{a}+\epsilon_{a b c} a_{b} \dot{a}_{c}\right),
$$

with $a_{4}^{2}+\sum_{a=1}^{3} a_{a}^{2}=1$ parametrizing the $\mathrm{SU}(2) \sim S^{3}$ moduli. Thus, the canonical momenta for $y_{I}$ read

$$
\begin{aligned}
& \Pi_{a}=m_{y} \rho^{2} \dot{a}_{a}-\frac{2 c_{3}}{m_{H} \pi^{2} a \rho^{2}}\left(a_{4} \chi^{\dagger} \tau_{a} \chi+\epsilon_{c b a} \chi^{\dagger} \tau_{c} \chi a_{b}\right), \\
& \Pi_{4}=m_{y} \rho^{2} \dot{a}_{4}+\frac{2 c_{3}}{m_{H} \pi^{2} a \rho^{2}} \chi^{\dagger} \tau_{a} \chi a_{a} .
\end{aligned}
$$

Therefore the spin-orbit contribution to the Hamiltonian is

$$
\begin{aligned}
H & =\frac{1}{2 m_{y} \rho^{2}}\left(-i \nabla_{\mathrm{S}^{3}}+\frac{4 c_{3}}{m_{H} \pi^{2} a \rho^{2}} \vec{r} \times \vec{S}\right)^{2} \\
& =\frac{-\nabla_{\mathrm{S}_{3}}^{2}}{2 m_{y} \rho^{2}}+\frac{8 c_{3}}{m_{H} \pi^{2} a \rho^{2}} \frac{\vec{L} \cdot \vec{S}}{m_{y} \rho^{2}}+\left(\frac{4 c_{3}}{m_{H} \pi^{2} a \rho^{2}}\right)^{2} \frac{\chi^{\dagger} \frac{\vec{\tau}}{2} \chi \cdot \chi^{\dagger} \frac{\vec{\tau}}{2} \chi}{2 m_{y} \rho^{2}},
\end{aligned}
$$

with the orbital angular momentum

$$
\vec{L}=\frac{1}{2}\left(i \vec{a} \nabla_{4}-i a_{4} \vec{\nabla}-i \vec{a} \times \vec{\nabla}\right) .
$$

The integer-valued spin of the heavy-light doublet translates to a half-integer spin on the moduli

$$
\vec{S}=\chi^{\dagger} \frac{\vec{\tau}}{2} \chi,
$$

a nontrivial transmutation induced by the binding of the zero mode to the instanton in bulk [28]. To leading order in $1 / m_{H}$ only the first two contributions in Eq. (32) will be retained. The last contribution in Eq. (32) is the induced spin-spin interaction of the heavy mesons and is suppressed by $1 / m_{H}^{2}$.

\section{B. Spin effects}

The leading spin effects to order $1 / m_{H}$ stem from the quadratic and quartic $\chi$ contributions detailed above. The terms with a first-order time derivative of $\chi$ are

$$
\left(1+\frac{3 N_{c}}{2 m_{H} m_{y} \rho^{2}}\right) i \chi^{\dagger} \dot{\chi}+\frac{\dot{\chi}^{\dagger} \dot{\chi}}{2 m_{H}}-i \frac{2 \chi^{\dagger} \chi\left(\chi^{\dagger} \dot{\chi}-\dot{\chi}^{\dagger} \chi\right)}{3 m_{H} m_{y} \rho^{2}},
$$

and imply the equation of motion

$$
\begin{aligned}
- & \frac{1}{2 m_{H}} \partial_{t}^{2} \chi+i\left(1+\frac{3 N_{c}}{2 m_{H} m_{y} \rho^{2}}\right) \partial_{t} \chi+\frac{3 N_{c}}{2 m_{y} \rho^{2}} \chi \\
+ & \frac{4 i\left(\chi^{\dagger} \chi \dot{\chi}+\chi^{\dagger} \dot{\chi} \chi\right)}{3 m_{H} m_{y} \rho^{2}}=0 .
\end{aligned}
$$

Therefore to second order in $1 / m_{H}$ one has

$$
\begin{aligned}
i \partial_{t} \chi= & -\frac{3 N_{c}}{2 m_{y} \rho^{2}} \chi+\frac{9 N_{c}^{2}}{8 m_{H} m_{y}^{2} \rho^{4}} \chi+\frac{4 \chi^{\dagger} \chi \chi N_{c}}{m_{H} m_{y}^{2} \rho^{4}} \\
& +\frac{81 N_{c}^{3}}{32 m_{H}^{2} m_{y}^{3} \rho^{6}} \chi-\frac{32 N_{c}\left(\chi^{\dagger} \chi\right)^{2} \chi}{3 m_{H}^{2} m_{y}^{2} \rho^{6}}
\end{aligned}
$$

from which the Hamiltonian can be easily extracted.

\section{Hamiltonian}

With the above in mind and to obtain the Hamiltonian in leading order in $1 / m_{H}$, it is sufficient to perform the following substitution:

$$
j \rightarrow-\frac{3 N_{c}}{2 \tilde{\rho}^{2}},
$$

and add

$$
\delta H=\frac{81}{8 m_{H} \tilde{\rho}^{4}} n+\frac{1}{m_{H}^{2} \tilde{\rho}^{6}}\left(\frac{81 N_{c}^{3}}{32} n-\frac{32 N_{c}}{9} n^{3}\right)
$$

to the spin-independent Hamiltonian [28].

More specifically, for a single heavy quark with $n=1$, the total Hamiltonian to order $1 / m_{H}$ now reads $\left(N_{c}=3\right)$

$$
\begin{aligned}
H_{\text {single }}= & \frac{39 \vec{L} \cdot \vec{S}}{5 m_{H} m_{y}^{2}\left(1+\frac{1}{2 m_{H} m_{y} \rho^{2}}\right) \rho^{4}} \\
& +\left(-\frac{553}{120 m_{H} m_{y}^{2} \rho^{4}}+\frac{67.94}{m_{H}^{2} m_{y}^{4} \rho^{6}}+\frac{37+12 \frac{Z^{2}}{\rho^{2}}}{192 m_{H}}\right) \\
& -\frac{1}{2 m_{y} \rho^{3}\left(1+\frac{1}{2 m_{H} m_{y} \rho^{2}}\right)^{2}} \frac{\partial}{\partial \rho}\left(\rho^{3}\left(1+\frac{1}{2 m_{H} m_{y} \rho^{2}}\right) \frac{\partial}{\partial \rho}\right) \\
& +\frac{4 \vec{L}^{2}}{2 m_{y} \rho^{2}\left(1+\frac{1}{2 m_{H} m_{y} \rho^{2}}\right)} .
\end{aligned}
$$

The change in the Laplacian is due to the $\frac{\dot{\rho}^{2}}{\rho^{2}}+a_{I}^{2}$ term following from the new line element on the moduli,

$$
d s^{2}=\left(1+\frac{1}{2 m_{H} m_{y} \rho^{2}}\right) d y_{I}^{2},
$$

with a change in the small $\rho$ behavior. For the pentaquark states where $N_{\bar{Q}}=N_{Q}=1$, the corresponding Hamiltonian is $\left(N_{c}=3\right)$ 


$$
\begin{aligned}
H_{\text {double }}= & \frac{39 \vec{L} \cdot \vec{S}}{5 m_{H} m_{y}^{2}\left(1+\frac{1}{m_{H} m_{y} \rho^{2}}\right) \rho^{4}} \\
& +\left(-\frac{411}{20 m_{H} m_{y}^{2} \rho^{4}}+\frac{133.30}{m_{H}^{2} m_{y}^{4} \rho^{6}}+\frac{37+12 \frac{Z^{2}}{\rho^{2}}}{96 m_{H}}\right) \\
& -\frac{1}{2 m_{y} \rho^{3}\left(1+\frac{1}{m_{H} m_{y} \rho^{2}}\right)^{2}} \frac{\partial}{\partial \rho}\left(\rho^{3}\left(1+\frac{1}{m_{H} m_{y} \rho^{2}}\right) \frac{\partial}{\partial \rho}\right) \\
& +\frac{4 \vec{L}^{2}}{2 m_{y} \rho^{2}\left(1+\frac{1}{m_{H} m_{y} \rho^{2}}\right)} .
\end{aligned}
$$

Below we solve the corresponding Schrödinger equation numerically.

\section{INDUCED QUANTUM POTENTIALS}

\section{A. The effective potential for a single-heavy quark: $l=0$ state}

For $l=0$, the spin-orbit coupling vanishes, i.e., $\vec{L} \cdot \vec{S}=0$, and the induced effective potential simplifies to
$V(\rho)=\frac{m_{y} \omega_{\rho}^{2}}{2} \rho^{2}-\frac{7}{30 m_{y} \rho^{2}}+\left(-\frac{553}{120 m_{H} m_{y}^{2} \rho^{4}}+\frac{67.94}{m_{H}^{2} m_{y}^{4} \rho^{6}}\right)$.

Although the sign of the $\frac{1}{\rho^{2}}$ is negative, the $m_{H}=\infty$ system is still stable due to the uncertainty principle. Indeed, for small $\rho$, the kinetic contribution is of order $\frac{1}{\rho^{2}}$ and compensates for the negative sign to maintain stability. In this case, the $1 / m_{H}$ term implies additional repulsion that further stabilizes the system. As $m_{H} \rightarrow \infty$, the spectrum approaches the infinite mass limit smoothly.

\section{B. The effective potential for a single-heavy quark: $\boldsymbol{l}>\mathbf{0}$ state}

For $l=2,4, \ldots$, one has $J=(l \pm 1) / 2$. We first consider the $J=(l-1) / 2$ case. Again for $N_{Q}=1$ and $N_{c}=3$, the effective potential reads

$$
\begin{aligned}
V\left(J=\frac{l-1}{2}, \rho\right)= & \frac{1}{2 m_{y}\left(1+\frac{1}{2 m_{H} m_{y} \rho^{2}}\right) \rho^{2}}\left(l(l+2)-\frac{(l+2) \alpha N_{c}}{m_{H} m_{y} \rho^{2}}+\frac{3 \alpha^{2} N_{c}^{2}}{4 m_{H}^{2} m_{y}^{2} \rho^{4}}\right) \\
& +\frac{m_{y} \omega_{\rho}^{2}}{2} \rho^{2}-\frac{7}{30 m_{y} \rho^{2}}+\left(-\frac{553}{120 m_{H} m_{y}^{2} \rho^{4}}+\frac{67.94}{m_{H}^{2} m_{y}^{4} \rho^{6}}\right)
\end{aligned}
$$

with $\alpha=\frac{13}{10}$. The $1 / m_{H}^{2}$ term due to the spin-orbit coupling is kept to maintain stability at small $\rho$. The change of the potential as one increases $m_{H}$ tends to decrease for larger $l$. For $l=2$, the shapes of the potential at $m_{H}=2$ and $m_{H}=\infty$ differ moderately, but for $l=2$ the difference is already quite small.

Similarly, in the $J=\frac{l+1}{2}$ case the effective potential is

$$
\begin{aligned}
V\left(J=\frac{l+1}{2}, \rho\right)= & \frac{1}{2 m_{y}\left(1+\frac{1}{2 m_{H} m y \rho^{2}}\right) \rho^{2}}\left(l(l+2)+\frac{l \alpha N_{c}}{m_{H} m_{y} \rho^{2}}+\frac{3 \alpha^{2} N_{c}^{2}}{4 m_{H}^{2} m_{y}^{2} \rho^{4}}\right) \\
& +\frac{m_{y} \omega_{\rho}^{2}}{2} \rho^{2}-\frac{7}{30 m_{y} \rho^{2}}+\left(-\frac{553}{120 m_{H} m_{y}^{2} \rho^{4}}+\frac{67.94}{m_{H}^{2} m_{y}^{4} \rho^{6}}\right) .
\end{aligned}
$$

Again, the $1 / m_{H}$ contribution further stabilizes the system and pushes the spectrum a little bit higher.

\section{The effective potential for a pentaquark state}

Here we focus on the pentaquark states with $N_{Q}=$ $\bar{N}_{Q}=1$ state or hidden $Q=c, b$, with $S=0,1$. For $S=0$, the potential reads

$$
\begin{aligned}
V\left(J=\frac{l}{2}, S=0\right)= & \frac{m_{y} \omega_{\rho}^{2} \rho^{2}}{2}+\frac{18}{5 m_{y} \rho^{2}}+\frac{l(l+2)}{2 m_{y}\left(1+\frac{1}{m_{H} m_{y}^{2} \rho^{2}}\right) \rho^{2}} \\
& +\left(-\frac{411}{20 m_{H} m_{y}^{2} \rho^{4}}+\frac{133.30}{m_{H}^{2} m_{y}^{4} \rho^{6}}\right)
\end{aligned}
$$

For $S=1$ we can have $J=l-1, l, l+1$, and the potential in this case reads 


$$
\begin{aligned}
V(J, S=1, \rho)= & \frac{1}{2 m_{y}\left(1+\frac{1}{m_{H} m_{y}^{2} \rho^{2}}\right) \rho^{2}}\left(l(l+2)+\frac{2 N_{c} \Delta(J) \beta}{m_{H} m_{y} \rho^{2}}+\frac{2 N_{c}^{2} \beta^{2}}{m_{H}^{2} m_{y}^{2} \rho^{4}}\right)+\frac{18}{5 m_{y} \rho^{2}} \\
& +\frac{m_{y} \omega_{\rho}^{2}}{2} \rho^{2}+\left(-\frac{411}{20 m_{H} m_{y}^{2} \rho^{4}}+\frac{133.30}{m_{H}^{2} m_{y}^{4} \rho^{6}}\right),
\end{aligned}
$$

with $\beta=\frac{13}{10}$ and

$$
\Delta(J)=J(J+1)-\frac{l(l+2)}{4}-2 .
$$

More specifically, for $l=1$ we have $\Delta(J=1 / 2)=-2$ and $\Delta(J=3 / 2)=1$.

\section{SPECTRA}

Given the Hamiltonian and the explicit induced quantum potentials, we can now obtain the spectra of the holographic heavy-light hadrons. Our strategy is the following: we treat the warping contribution as a small perturbation, while solving the radial part numerically. For the warping part, using the average

$$
\left\langle\frac{Z^{2}}{\rho^{2}}\right\rangle_{n_{z}, n_{\rho}}=\frac{n_{z}+\frac{1}{2}}{\tilde{l}+1}
$$

we obtain

$$
\delta M_{\mathrm{warp}}=\frac{37+\frac{6}{\tilde{l}+1}}{192 m_{H}}\left(N_{Q}+N_{\bar{Q}}\right)
$$

in units of $M_{K K}$.

To obtain the radial part, we need to solve the Schrödinger equation

$$
\begin{aligned}
& -\frac{1}{2 m_{y} \rho^{3}\left(1+\frac{1}{2 m_{H} m_{y} \rho^{2}}\right)^{2}} \frac{\partial}{\partial \rho}\left(\rho^{3}\left(1+\frac{1}{2 m_{H} m_{y} \rho^{2}}\right) \frac{\partial}{\partial \rho}\right) \Psi_{n, l}(\rho) \\
& +V_{l}(\rho) \Psi_{n, l}(\rho)=E_{n, l} \Psi_{n, l}(\rho)
\end{aligned}
$$

with the warped normalization condition

$$
2 \pi^{2} \int_{0}^{\infty} \rho^{3}\left(1+\frac{1}{2 m_{y} \rho^{2}}\right)^{2}\left|\Psi_{n, l}(\rho)\right|^{2} d \rho=1 .
$$

For this purpose we perform the transformation $\Psi \rightarrow u$ and use $\tilde{\rho}^{2}=m_{y} \rho^{2}$,

$$
\Psi=\frac{u}{\sqrt{\rho^{3}\left(1+\frac{1}{2 m_{H} m_{y} \rho^{2}}\right)}},
$$

to simplify Eq. (51) as

$$
\begin{aligned}
& -\frac{1}{2\left(1+\frac{1}{2 m_{H} \tilde{\rho}^{2}}\right)} u_{n, l}^{\prime \prime}(\tilde{\rho})+\left[\frac{3\left(1+\frac{1}{m_{H} \rho^{2}}-\frac{1}{12 m_{H}^{2} \tilde{\rho}^{4}}\right)}{8 \tilde{\rho}^{2}\left(1+\frac{1}{2 m_{H} \tilde{\rho}^{2}}\right)}\right] u_{n, l}(\tilde{\rho}) \\
& \quad+V_{l}(\tilde{\rho}) u_{n, l}(\tilde{\rho})=E_{n, l} u_{n, l}(\tilde{\rho}),
\end{aligned}
$$

with the normalization condition

$$
2 \pi^{2} \int_{0}^{\infty} d \tilde{\rho}\left(1+\frac{1}{2 m_{H} \tilde{\rho}^{2}}\right)\left|u_{n, l}(\tilde{\rho})\right|^{2}=1
$$

Notice that the normalization condition actually requires the $u_{n, l}$ to vanish near $\tilde{\rho}=0$. In this case one can show that although the additional term [large bracket in Eq. (51)] becomes negative at small $\tilde{\rho}$, the spectrum $E_{n, l}$ is still bounded from below. The above equation for $u_{n, l}$ can be diagonalized numerically and below we present the results for different states.

To fix the parameters for the charmed heavy baryons, we choose $M_{D}=1.87 \mathrm{GeV}$ for the D-meson mass in Eq. (7) and fix $M_{K K}=0.475 \mathrm{GeV}$ to reproduce the $M_{\Lambda_{c}}=2.286 \mathrm{GeV}$. This low value of $M_{K K}$ is consistent with the value used to reproduce the nucleon spectra [32], but about half the value of $M_{K K} \sim 1 \mathrm{GeV}$ used originally in Eq. [26] and adopted in [3,28,29]. In this case we have $m_{H}=(1.87-0.168) \mathrm{GeV}=3.66 M_{K K}$. In Fig. 1 we show the radial wave functions for the first and second excited states following from Eq. (51) for a single heavy-baryon (top panel) and double-heavy-baryon or pentaquark state (bottom panel). Note the rapid decay of the wave functions near the instanton core as $\rho \rightarrow 0$.

The corresponding charm and bottom states for singleand double-heavy hadrons are listed in Tables I and II respectively. Note that while $m_{\Lambda_{c}}=2.286 \mathrm{GeV}$ is fitted to fix the Kaluza-Klein scale $M_{K K}=0.475 \mathrm{GeV}, m_{\Lambda_{b}}=$ $5.608 \mathrm{GeV}$ is a holographic prediction which is remarkably close to the experimental value of $5.620 \mathrm{GeV}$. The details of the mass budgets for each of the states in terms of the three holographic parameters are given in Appendix C. The results for the single-heavy baryon spectrum are surprisingly good, given the small number of parameters used in this holographic approach. The spin contributions improve considerably the predictions for the masses and their hierarchy. In particular, the empirical mass ordering $\Sigma_{c}-$ $\Lambda_{c}<\Lambda_{c}^{*}-\Lambda_{c}$ is obtained, contrary to the claim in [31]. The mass splitting between $\Sigma_{c}$ and $\Sigma_{b}$ is higher than observed due to the sizable repulsion from the $l=2$ intrinsic angular momentum assignment. 

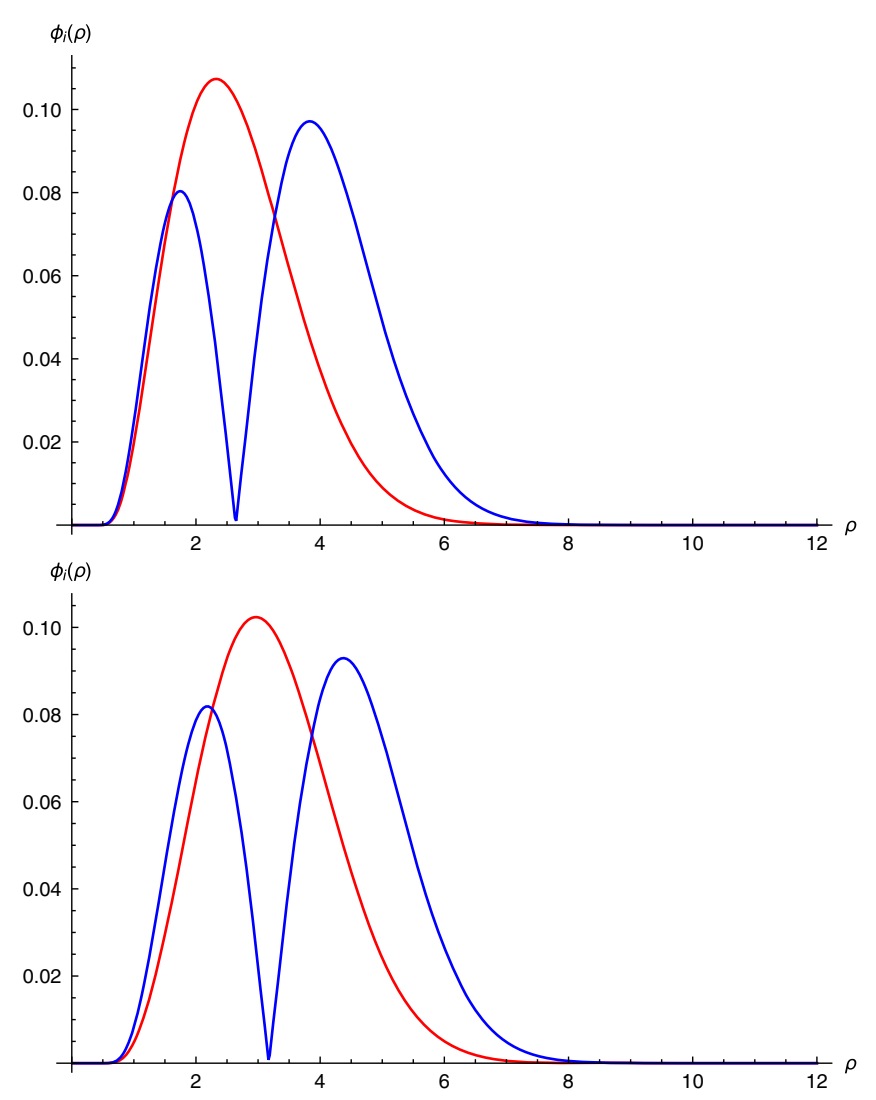

FIG. 1. The $l=0$ radial wave functions for single heavybaryon (top panel) and double-heavy-baryon or pentaquark state (bottom panel) as a function of $\tilde{\rho}$. See text.

The holographic construction with spin corrections allows for only three pentaquark states which are close to the observed charmed pentaquark states reported by $\mathrm{LHCb}$, although with slightly smaller masses (the $80 \mathrm{MeV}$ difference can be easily narrowed by adjusting the KaluzaKlein scale $M_{K K}=0.475 \mathrm{GeV}$ at the expense of $\Lambda_{c}$ ). The spin-orbit effects split away the $\left[\frac{1}{2} \frac{1}{2}^{-}\right]_{S=1}$ and $\left[\frac{1}{2}^{2}\right]_{S=1}$ states, lifting the degeneracy reported originally in [3]. The present holographic construction rules out a pentaquark with a $\left[\frac{1}{2} \frac{5}{2}\right]$ assignment since the instanton core carries equal spin and isospin [3]. The splittings between the different pentaquark states are somehow smaller than expected, due to the strength of the spin-orbit coupling to order $1 / m_{H}^{2}$. Additional contributions are expected to order $1 / m_{H}^{3}$. This construction supports additional Roper-like and odd-parity-like pentaquark states which we have denoted by $P_{c, b}^{*}$, although they are heavier and more susceptible to decay.

\section{CONCLUSIONS}

In the holographic construction presented in [3,28,29], heavy hadrons are described in bulk using a set of degenerate $N_{f}$ light D8-D $\overline{8}$ branes plus one heavy probe brane in the cigar-shaped geometry that spontaneously breaks chiral symmetry. This construction enforces both chiral and heavy-quark symmetry and describes well the low-lying heavy-light mesons and baryons. Heavy baryons are composed of heavy-light mesons bound to a core instanton in bulk. Remarkably, the bound heavy-light mesons with spin 1 transmute to heavy quarks with spin $\frac{1}{2}$, an amazing spin-statistics transmutation by geometry.

In $[3,28,29]$ the analysis of the bound states and spectra was carried to order $m_{H}^{0}$ where the spin effects are absent. In this work and for $N_{f}=2$, we have now carried the analysis at next to leading order in $1 / m_{H}$ where the spinorbit and spin corrections are manifest. By refining the Kaluza-Klein scale $M_{K K}$ from $1 \mathrm{GeV}$ used in $[3,29]$ to $0.475 \mathrm{GeV}$ used here, a rich spectrum with single- and double-heavy baryons emerges with fair agreement with the empirically observed states, including the newly reported charm pentaquark states by LHCb.

This is remarkable, given that only three parameters were used in the holographic construction: $M_{0}, M_{K K}, m_{H}$. For charm, they are fixed by $M_{0} \rightarrow m_{N}$ (nucleon mass), $M_{K K} \rightarrow M_{\Lambda_{c}}$ (lambda mass) and $m_{H} \rightarrow M_{D}$ (D-meson mass). The only parameter adjustment for the bottom spectrum is $m_{H} \rightarrow M_{B}$ (B-meson mass). Needless to say,

TABLE I. Charm baryons and pentaquarks.

\begin{tabular}{ccccccccc}
\hline \hline$B$ & $I J^{P}$ & $l$ & $n_{\rho}$ & $n_{z}$ & $N_{Q}$ & $N_{\bar{Q}}$ & Mass $(\mathrm{MeV})$ & Experiment $(\mathrm{MeV})$ \\
\hline$\Lambda_{c}$ & $0 \frac{1}{2}^{+}$ & 0 & 0 & 0 & 1 & 0 & 2286 & 2286 \\
$\Sigma_{c}$ & $1 \frac{1}{2}$ & 2 & 0 & 0 & 1 & 0 & 2557 & 2453 \\
& $1 \frac{3}{2}^{+}$ & 2 & 0 & 0 & 1 & 0 & 2596 & 2520 \\
$\Lambda_{c}^{*}$ & $0 \frac{1}{2}$ & 0 & 0 & 1 & 1 & 0 & 2683 & 2595 \\
& $0 \frac{1}{2}^{+}$ & 0 & 1 & 0 & 1 & 0 & 2726 & 2765 \\
$\Sigma_{c}^{*}$ & $1 \frac{1}{2}^{-}, 1 \frac{3}{2}-$ & 2 & 0 & 1 & 1 & 0 & {$[2947 / 2986]$} & $\ldots$ \\
& $1 \frac{1}{2}^{+}, 1 \frac{3}{2}+$ & 2 & 1 & 0 & 1 & 0 & {$[2948 / 2995]$} & $\ldots$ \\
$P_{c}$ & $1^{2} \frac{1}{2}, \frac{13}{2} \frac{3}{2}^{-}$ & 1 & 0 & 0 & 1 & 1 & {$[4340 / 4360 / 4374]$} & {$[4312 / 4440 / 4457]$} \\
$P_{c}^{*}$ & $1 \frac{1}{2}^{-}, 1 \frac{3}{2}$ & 1 & 0 & 1 & 1 & 1 & {$[4732 / 4752 / 4767]$} & $\ldots$ \\
& $1 \frac{1}{2}^{+}, 1 \frac{3}{2}+$ & 1 & 1 & 0 & 1 & 1 & {$[4725 / 4746 / 4763]$} & $\ldots$ \\
\hline \hline
\end{tabular}


TABLE II. Bottom baryons and pentaquarks.

\begin{tabular}{ccccccccc}
\hline \hline$B$ & $I J^{P}$ & $l$ & $n_{\rho}$ & $n_{z}$ & $N_{Q}$ & $N_{\bar{Q}}$ & Mass $(\mathrm{MeV})$ & Experiment $(\mathrm{MeV})$ \\
\hline$\Lambda_{b}$ & $0 \frac{1}{2}^{+}$ & 0 & 0 & 0 & 1 & 0 & 5608 & 5620 \\
$\Sigma_{b}$ & $1 \frac{1}{2}^{+}$ & 2 & 0 & 0 & 1 & 0 & 5962 & 5810 \\
& $1 \frac{3}{2}^{+}$ & 2 & 0 & 0 & 1 & 0 & 5978 & 5830 \\
$\Lambda_{b}^{*}$ & $0 \frac{1}{2}^{-}$ & 0 & 0 & 1 & 1 & 0 & 5998 & 5912 \\
& $0 \frac{1}{2}^{+}$ & 0 & 1 & 0 & 1 & 0 & 6029 & $(6072)$ \\
$\Sigma_{b}^{*}$ & $1 \frac{1}{2}, 1 \frac{3}{2}^{-}$ & 2 & 0 & 1 & 1 & 0 & {$[6351 / 6367]$} & $\ldots$ \\
& $1 \frac{1}{2}{ }^{+}, 1 \frac{3}{2}^{+}$ & 2 & 1 & 0 & 1 & 0 & {$[6344 / 6367]$} & $\ldots$ \\
$P_{b}$ & $\frac{1}{2}^{2}{ }^{-}, \frac{1}{2} \frac{3}{2}^{-}$ & 1 & 0 & 0 & 1 & 1 & {$[11155 / 11163 / 11167]$} & $\ldots$ \\
$P_{b}^{*}$ & $1 \frac{1}{2}{ }^{-}, 1 \frac{3}{2}^{-}$ & 1 & 0 & 1 & 1 & 1 & {$[11544 / 11553 / 11556]$} & $\ldots$ \\
& $1 \frac{1}{2}{ }^{+}, 1 \frac{3}{2}^{+}$ & 1 & 1 & 0 & 1 & 1 & {$[11532 / 11543 / 11579]$} & $\ldots$ \\
\hline \hline
\end{tabular}

the light-light, heavy-light and heavy-heavy mesons and baryons are described simultaneously, without changing the number of parameters.

The holographic construction predicts a triplet of nearly degenerate charm pentaquark states with the isospin-spin-parity assignments,

$$
\left(\left[P_{c}(4340) \frac{1}{2} \frac{1-}{2}\right]_{S=1},\left[P_{c}(4360) \frac{1}{2} \frac{1^{-}}{2}\right]_{S=0},\left[P_{c}(4374) \frac{1}{2} \frac{3^{-}}{2}\right]_{S=1}\right),
$$

which are to be compared to $P_{c}[4312|4440| 4457]$ recently reported by LHCb. The small mass discrepancy can be readily eliminated by adjusting the Kaluza-Klein scale at the expense of $\Lambda_{c}$. The spin-orbit effects split away the states with intrinsic spin $S=1$. The analysis rules out the assignment $\left[\frac{1}{2} \frac{5}{2}^{ \pm}\right]$for these states, and predicts a triplet of bottomed pentaquark states,

$$
\left(\left[P_{b}(11532) \frac{1}{2} \frac{1^{-}}{2}\right]_{S=1},\left[P_{b}(11543) \frac{1}{2} \frac{1^{-}}{2}\right]_{S=0},\left[P_{b}(11579) \frac{1}{2} \frac{3^{-}}{2}\right]_{S=1}\right)
$$

not yet observed. New Roper-like and odd-parity pentaquark states are also suggested, although much heavier and more susceptible to falling apart.

The recently reported $P_{c}(4337)$ at $3 \sigma$ significance [43] is not supported by our current holographic analysis of the low-lying pentaquark states. The even- and odd-parity excited holographic pentaquark states $P_{c}^{*}$ are heavier. More statistics to improve the significance of this pentaquark would be welcome.

Finally, the present holographic description can be regarded as the holographic dual of the chiral soliton construction of heavy-light baryons (see $[39,44]$ and references therein). However, in the latter the uncertainties in combining chiral and heavy quark symmetry strongly limit their predictive range, especially when one addresses the spin corrections. This is not the case for the holographic description as we have shown, as both symmetries are geometrically embedded in the bulk brane construction with just three parameters. The dual approach is vastly superior.

\section{ACKNOWLEDGMENTS}

This work is supported by the Office of Science, U.S. Department of Energy, under Contract No. DE-FG88ER40388 and by Polish National Science Centre (NCN) Grant No. UMO-2017/27/B/ST2/01139.

Note added.-We should also mention that our setup allows the determination of not only the masses of the pentaquarks, but also their decay widths. This will be explored in part II [45] and part III [46] of the series of papers.

\section{APPENDIX A: DETAILS OF THE HEAVY MASS EXPANSION}

Following the rescaling in Eq. (9) the effective action for the heavy-light fields split into the following contributions:

$$
\mathcal{L}=a N_{c} \lambda \mathcal{L}_{0}+a N_{c}\left(\mathcal{L}_{1}+\tilde{\mathcal{L}}_{1}\right)+\mathcal{L}_{\mathrm{CS}}
$$

with each contribution given by 


$$
\begin{aligned}
\mathcal{L}_{0}= & -\left(D_{M} \Phi_{N}^{\dagger}-D_{N} \Phi_{M}^{\dagger}\right)\left(D_{M} \Phi_{N}-D_{N} \Phi_{M}\right) \\
& +2 \Phi_{M}^{\dagger} F_{M N} \Phi_{N}, \\
\mathcal{L}_{1}= & +2\left(D_{0} \Phi_{M}^{\dagger}-D_{M} \Phi_{0}^{\dagger}\right)\left(D_{0} \Phi_{M}-D_{M} \Phi_{0}\right)-2 \Phi_{0}^{\dagger} F^{0 M} \Phi_{M} \\
& -2 \Phi_{M}^{\dagger} F^{M 0} \Phi_{0}-2 m_{H}^{2} \Phi_{M}^{\dagger} \Phi_{M}, \\
\tilde{\mathcal{L}}_{1}= & +\frac{z^{2}}{3}\left(D_{i} \Phi_{j}-D_{j} \Phi_{i}\right)^{\dagger}\left(D_{i} \Phi_{j}-D_{j} \Phi_{i}\right) \\
& -2 z^{2}\left(D_{i} \Phi_{z}-D_{z} \Phi_{i}\right)^{\dagger}\left(D_{i} \Phi_{z}-D_{z} \Phi_{i}\right) \\
& -\frac{2}{3} z^{2} \Phi_{i}^{\dagger} F_{i j} \Phi_{j}+2 z^{2}\left(\Phi_{z}^{\dagger} F_{z i} \Phi_{i}+\text { c.c. }\right) \\
\mathcal{L}_{\mathrm{CS}}= & -\frac{i N_{c}}{16 \pi^{2}} \Phi^{\dagger}\left(d A+A^{2}\right) D \Phi \\
& -\frac{i N_{c}}{16 \pi^{2}}(D \Phi)^{\dagger}\left(d A+A^{2}\right) \Phi+\mathcal{O}\left(\Phi^{3}\right) .
\end{aligned}
$$

We now use the expansion (17) to explicitly derive the various contributions in Eq. (A1) in leading order in $1 / m_{H}$. The net result has manifest heavy quark symmetry to order $m_{H}^{0}$, with the spin-orbit and spin-spin contributions breaking this symmetry to order $1 / m_{H}$.

\section{Kinetic contribution: $\mathcal{L}_{\text {kin }}$}

The explicit form of the kinetic contribution is

$\mathcal{L}_{\text {kin }}=\frac{1}{8 m_{H}} \chi^{\dagger} \sigma_{M} f\left(-\partial_{t}-\hat{A}_{0}-\Phi\right)\left(\partial_{t}+\hat{A}_{0}+\Phi\right) \bar{\sigma}_{M} f \chi$,

which contains a derivative of $\chi$. With the help of the identity for Weyl matrices $\sigma_{M} \tau^{a} \bar{\sigma}_{M}=0$, Eq. (A3) reads

$$
\begin{aligned}
& \frac{1}{2 m_{H}} f^{2} \dot{\chi}^{\dagger} \dot{\chi}+\frac{1}{2 m_{H}} \dot{X}_{N} \dot{X}_{M} \partial_{N} f \partial_{M} f \chi^{\dagger} \chi+\frac{f^{2}}{2 m_{H}} \hat{A}_{0}\left(\dot{\chi}^{\dagger} \chi-\chi^{\dagger} \dot{\chi}\right) \\
& -\frac{f^{2}}{2 m_{H}} \hat{A}_{0}^{2} \chi^{\dagger} \chi-\frac{f^{2}}{4 m_{H}} \operatorname{tr}(\Phi)^{2} \chi^{\dagger} \chi,
\end{aligned}
$$

which can be further simplified by using the explicit relations

$$
\begin{aligned}
-\operatorname{tr} \Phi^{2} & =\frac{X^{4}}{4\left(X^{2}+\rho^{2}\right)^{2}} 2 \chi^{a \dagger} \chi^{a}-\dot{X}_{N} \dot{X}_{M} \operatorname{tr} A_{N} A_{M} \\
& =\frac{2 X^{4}}{\left(X^{2}+\rho^{2}\right)^{2}} \dot{a}_{I}^{2}+\frac{3 X^{2}}{2\left(X^{2}+\rho^{2}\right)^{2}} \dot{X}^{2}
\end{aligned}
$$

and

$$
\hat{A}_{0}=-\frac{i}{8 \pi^{2} a x^{2}}\left(1-\frac{\rho^{4}}{\left(x^{2}+\rho^{2}\right)^{2}}\right)
$$

to have

$$
\begin{aligned}
\mathcal{L}_{\mathrm{kin}}= & \frac{\dot{\chi}^{\dagger} \dot{\chi}}{2 m_{H}}+\left(\frac{1}{4 m_{H}} \frac{\dot{\rho}^{2}}{\rho^{2}}+\frac{\dot{a}_{I}^{2}}{4 m_{H}}+\frac{\dot{X}^{2}}{4 m_{H} \rho^{2}}\right) \chi^{\dagger} \chi \\
& +\frac{1}{16 m_{H} \pi^{2} a \rho^{2}} i \chi^{\dagger} \partial_{t} \chi+\frac{13}{3840 m_{H} \pi^{4} a^{2} \rho^{4}} \chi^{\dagger} \chi
\end{aligned}
$$

after integration over space.

\section{Chern-Simons contribution: $\mathcal{L}_{\mathrm{CS}}$}

The Chern-Simons term is

$$
\begin{aligned}
\mathcal{L}_{\mathrm{CS}} & =-\frac{i N_{c}}{16 \pi^{2}} \Phi^{\dagger}\left(d A+A^{2}\right) D \Phi-\frac{i N_{c}}{16 \pi^{2}}(D \Phi)^{\dagger}\left(d A+A^{2}\right) \Phi \\
& =-\frac{i N_{c}}{8 \pi^{2}} \Phi^{\dagger}\left(d A+A^{2}\right) D \Phi
\end{aligned}
$$

where on the second line we have performed a partial integration with the help of the Bianchi identity $D F=0$. More explicitly, we have

$$
\begin{aligned}
& \frac{i}{128 m_{H} \pi^{2} a} f^{2} \chi^{\dagger} \sigma_{M} F_{M N} \bar{\sigma}_{N} \dot{\chi} \\
& +\frac{i \epsilon_{M N P Q}}{128 m_{H} \pi^{2} a} f \chi^{\dagger} \sigma_{M} F_{0 N}\left(\partial_{P}+A_{P}\right) f \bar{\sigma}_{Q} \chi \\
& +\hat{A}_{0}\left(\frac{i N_{c}}{128 m_{H} \pi^{2} a} \chi^{\dagger} \sigma_{M} F_{M N} \bar{\sigma}_{N} \chi f^{2}\right),
\end{aligned}
$$

which is seen to contain $\chi^{\dagger} \chi$ as well as linear terms in derivatives. Recall that the electric field $F_{0 M}$ after solving for the Gauss constraint reads

$$
F_{0 M}=\dot{X}_{N} F_{M N}+\dot{\rho} \frac{\partial A_{M}}{\partial \rho}-\chi^{a} D_{M} \Phi^{a}
$$

The linear terms in $\dot{\rho}, \dot{X}_{N}$ vanish due to parity and translational invariance, but there are terms of the form

$$
\chi^{a} \chi^{\dagger} \frac{\tau^{a}}{2} \chi=i \chi^{\dagger} \mathbf{a}^{-1} \dot{\mathbf{a}} \chi
$$

which couple to isospin. Again, using the identity for Weyl matrices

$$
\epsilon_{M N P Q} \bar{\sigma}_{Q R}=-\delta_{M R} \bar{\sigma}_{M P}+\delta_{N R} \bar{\sigma}_{M P}-\delta_{P Q} \bar{\sigma}_{M N},
$$

all terms that require antisymmetrization to vanish,

$$
-\epsilon_{M N P Q} \sigma_{M} F_{0 N} \bar{\sigma}_{P} \partial_{Q} f=0
$$

but the more involved one 


$$
\begin{aligned}
- & \epsilon_{M N P Q} \sigma_{M} F_{0 N} A_{P} \bar{\sigma}_{Q} \\
= & \frac{\sigma_{M} F_{0 N} \bar{\sigma}_{N Q} \bar{\sigma}_{Q} X_{M}}{X^{2}+\rho^{2}}-\frac{\sigma_{M} F_{0 N} \bar{\sigma}_{M Q} \bar{\sigma}_{Q} X_{N}}{X^{2}+\rho^{2}} \\
& +\frac{\sigma_{M} F_{0 N} \bar{\sigma}_{M N} \bar{\sigma}_{Q} X_{Q}}{X^{2}+\rho^{2}}
\end{aligned}
$$

does not. Using the identity $\sigma_{M} \tau^{i} \bar{\sigma}_{M}=0$, the second term vanishes, while the first and the third term read

$$
\begin{aligned}
& \frac{\sigma_{M} F_{0 N} \bar{\sigma}_{N Q} \bar{\sigma}_{Q} X_{M}}{X^{2}+\rho^{2}}=\frac{3 \sigma \cdot X F_{0 N} \bar{\sigma}_{N}}{X^{2}+\rho^{2}}=-\frac{9 \rho^{2} f}{\left(X^{2}+\rho^{2}\right)^{2}} \tau^{a} \chi^{a}, \\
& \frac{\sigma_{M} F_{0 N} \bar{\sigma}_{M N} \bar{\sigma}_{Q} X_{Q}}{X^{2}+\rho^{2}}=-\frac{\sigma_{M} F_{0 N} \bar{\sigma}_{N} \sigma_{M} \bar{\sigma} \cdot X}{2\left(X^{2}+\rho^{2}\right)}=\frac{3 \rho^{2} f}{\left(X^{2}+\rho^{2}\right)^{2}} \tau^{a} \chi^{a} .
\end{aligned}
$$

Since

$$
-\epsilon_{M N P Q} \sigma_{M} F_{0 N} A_{P} \bar{\sigma}_{Q}=-\frac{6 \rho^{2} f}{\left(X^{2}+\rho^{2}\right)^{2}} \tau^{a} \chi^{a}
$$

we finally have

$$
-\epsilon_{M N P Q} \Phi_{M}^{\dagger} F_{0 N} D_{P} \Phi_{Q}=-\frac{6 \rho^{2} f^{2}}{16 m_{H} N_{c} a\left(X^{2}+\rho^{2}\right)^{2}} \chi^{\dagger} \tau^{a} \chi \chi^{a} .
$$

One should also consider the contribution from $\hat{F}_{0 N}=$ $-\frac{1}{r} \frac{\partial \hat{A}_{0}}{\partial r} x_{N}$,

$$
-\epsilon_{M N P Q} \sigma_{M} \hat{F}_{0 N} A_{P} \bar{\sigma}_{Q}=\frac{6 X^{2}}{X^{2}+\rho^{2}} \frac{1}{r} \frac{\partial \hat{A}_{0}}{\partial r} .
$$

The final Chern-Simons contribution to order $1 / m_{H}$ after rescaling is

$$
\begin{gathered}
\frac{3}{16 m_{H} \pi^{2} a} \frac{f^{2} \rho^{2}}{\left(X^{2}+\rho^{2}\right)^{2}} i \chi^{\dagger}\left(\partial_{t}+\hat{A}_{0}\right) \chi \\
+\frac{3}{64 m_{H} \pi^{2} a} \frac{\rho^{2} f^{2}}{\left(X^{2}+\rho^{2}\right)^{2}} \chi^{\dagger} \tau^{a} \chi i \chi^{a} \\
-\frac{3 i}{64 m_{H} \pi^{2} a} \frac{X^{2} f^{2}}{X^{2}+\rho^{2}} \frac{1}{r} \frac{\partial \hat{A}_{0}}{\partial r} \chi^{\dagger} \chi
\end{gathered}
$$

In fact, the first term can be obtained from the leading-order result by noticing that $\partial_{t} \rightarrow-i m_{H}+\partial_{t}$ and requiring gauge invariance. Using

$$
\hat{A}_{0}=-\frac{i}{8 \pi^{2} a x^{2}}\left(1-\frac{\rho^{4}}{\left(x^{2}+\rho^{2}\right)^{2}}\right)
$$

and performing the spatial integration we finally have

$$
\begin{aligned}
\mathcal{L}_{\mathrm{CS}}= & \frac{1}{32 m_{H} \pi^{2} a \rho^{2}} i \chi^{\dagger} \partial_{t} \chi+\frac{7}{1280 m_{H} \pi^{4} a^{2} \rho^{4}} \chi^{\dagger} \chi \\
& +\frac{1}{128 m_{H} \pi^{2} a \rho^{2}} \chi^{\dagger} \tau^{a} \chi i \chi^{a} .
\end{aligned}
$$

\section{The contribution: $\Phi_{\mathbf{0}}$}

This is the most difficult term to unravel to order $1 / m_{H}$. The equation of motion for $\Phi_{0}$ reads

$$
\begin{aligned}
& \left(-D_{M}^{2}+m_{H}^{2}\right) \Phi_{0}+2 F_{M 0} \Phi_{M}-\frac{i}{16 \pi^{2} a} F_{P Q}\left(\partial_{P}+A_{P}\right) \Phi_{Q} \\
& =0
\end{aligned}
$$

after using the self-dual condition for $F$. Using the standard relations for $\bar{\sigma}_{M N}$, we have for the last two contributions in Eq. (A22)

$$
F_{P Q} \partial_{P} \Phi_{Q}=\frac{6 \rho^{2}}{\left(X^{2}+\rho^{2}\right)^{2}} \frac{1}{r} \frac{d f}{d r} \bar{\sigma} \cdot X \chi
$$

$$
F_{P Q} A_{P} \Phi_{Q}=-\frac{6 \rho^{2}}{\left(X^{2}+\rho^{2}\right)^{3}} f \bar{\sigma} \cdot X \chi .
$$

For the first contribution in Eq. (A22) we have

$F_{M 0} \Phi_{M}=\frac{6 f}{\left(X^{2}+\rho^{2}\right)^{2}}\left(\rho^{2} \bar{\sigma} \cdot \dot{X}+\bar{\sigma} \cdot X \rho \dot{\rho}\right) \chi+\chi^{a} D_{M} \Phi^{a} \bar{\sigma}_{M} \chi f$,

with

$$
\Phi^{a}=\frac{1}{2\left(X^{2}+\rho^{2}\right)} \bar{\sigma} \cdot X \tau^{a} \sigma \cdot X,
$$

or more explicitly

$$
\chi^{a} D_{M} \Phi^{a} \bar{\sigma}_{M} \chi f=\frac{3 \rho^{2} f}{\left(X^{2}+\rho^{2}\right)^{2}} \bar{\sigma} \cdot X \tau^{a} \chi \chi^{a} .
$$

Inserting Eqs. (A27) and (A28) into Eq. (A22) we have

$$
\left(-D_{M}^{2}+m_{H}^{2}\right) \Phi_{0}+J_{0}=0,
$$

with

$$
\begin{aligned}
J_{0}= & \frac{12 f}{\left(X^{2}+\rho^{2}\right)^{2}}\left(\rho^{2} \bar{\sigma} \cdot \dot{X}+\bar{\sigma} \cdot X \rho \dot{\rho}\right) \chi+\frac{6 f \rho^{2}}{\left(X^{2}+\rho^{2}\right)^{2}} \bar{\sigma} \\
& \cdot X \tau^{a} \chi \chi^{a}+\frac{3 i}{2 \pi^{2} a} \frac{\rho^{2} f}{\left(X^{2}+\rho^{2}\right)^{3}} \bar{\sigma} \cdot X \chi+\frac{2 f}{r} \frac{\partial \hat{A}_{0}}{\partial r} \bar{\sigma} \cdot X \chi
\end{aligned}
$$


the source for $\Phi_{0}$. In this equation the Abelian part of $F_{N 0}$ has been included. Since

$$
\frac{1}{r} \frac{\partial \hat{A}_{0}}{\partial r}=\frac{i}{4 \pi^{2} a} \frac{1}{\left(X^{2}+\rho^{2}\right)^{2}}\left(1+\frac{2 \rho^{2}}{X^{2}+\rho^{2}}\right)
$$

one finally has

$$
\begin{aligned}
J_{0}= & \frac{12 f}{\left(X^{2}+\rho^{2}\right)^{2}}\left(\rho^{2} \bar{\sigma} \cdot \dot{X}+\bar{\sigma} \cdot X \rho \dot{\rho}\right) \chi+\frac{6 \rho^{2} f}{\left(X^{2}+\rho^{2}\right)^{2}} \bar{\sigma} \cdot X \tau^{a} \chi \chi^{a} \\
& +\frac{i}{2 \pi^{2} a} \frac{f}{\left(X^{2}+\rho^{2}\right)^{2}}\left(1+\frac{5 \rho^{2}}{X^{2}+\rho^{2}}\right) \bar{\sigma} \cdot X \chi . \quad \text { (A31) }
\end{aligned}
$$

In the large $m_{H}$ limit, the contribution to $\Phi_{0}$ is $1 / m_{H}^{2}$ suppressed compared to $\Phi_{M}$

$\Phi_{0}=-\frac{1}{-D_{M}^{2}+m_{H}^{2}} J_{0}=-\frac{1}{m_{H}^{2}} J_{0}+\mathcal{O}\left(\frac{1}{m_{H}^{4}}\right)$,

and can be neglected from the Lagrangian.

\section{4. $\Phi_{0}$ at $m_{H}=0$}

In the opposite limit of $m_{H}=0$, it is instructive to see how the field $\Phi_{0}$ solves the constraint equation. To solve Eq. (A28), we define the Green's function

$$
\begin{aligned}
G(X, Y) & \equiv \frac{1}{-D_{M}^{2}}(X, Y) \\
& =\frac{\rho^{2}+\bar{\sigma} \cdot X \sigma \cdot Y}{4 \pi^{2}\left(X^{2}+\rho^{2}\right)^{\frac{1}{2}}(X-Y)^{2}\left(Y^{2}+\rho^{2}\right)^{\frac{1}{2}}},
\end{aligned}
$$

in terms of which the solution can be written as

$$
\Phi_{0}(X)=-\int d^{4} Y G(X, Y) J_{0}(Y)
$$

To perform the integral one needs the following elementary integrals:

$$
\begin{aligned}
& \int d^{4} Y \frac{\rho^{2}+\bar{\sigma} \cdot X \sigma \cdot Y}{4 \pi^{2}(X-Y)^{2}\left(Y^{2}+\rho^{2}\right)^{1 / 2}} \frac{\bar{\sigma} \cdot Y}{\left(Y^{2}+\rho^{2}\right)^{n+3 / 2}} \\
& \equiv f_{n}\left(X^{2}, \rho^{2}\right) \bar{\sigma} \cdot X
\end{aligned}
$$

with

$$
\begin{aligned}
f_{n}\left(X^{2}, \rho^{2}\right)= & \frac{\Gamma(n)}{4 \Gamma(n+2)} \frac{1}{X^{2}}\left[\left(2-(n-2) \frac{\rho^{2}}{X^{2}}\right) \frac{\Delta\left(n-1, X^{2}\right)}{n-1}\right. \\
& \left.+\frac{1}{X^{2}} \Delta\left(n-2, X^{2}\right)\right]
\end{aligned}
$$

and

$$
\begin{aligned}
& \int d^{4} Y \frac{\rho^{2}+\bar{\sigma} \cdot X \sigma \cdot Y}{4 \pi^{2}(X-Y)^{2}\left(Y^{2}+\rho^{2}\right)^{1 / 2}} \frac{1}{\left(Y^{2}+\rho^{2}\right)^{n+3 / 2}} \\
& \equiv g_{n}\left(X^{2}, \rho^{2}\right),
\end{aligned}
$$

with

$$
g_{n}\left(X^{2}, \rho^{2}\right)=\frac{1}{4(n+1) X^{2}} \frac{\Delta\left(n-1, X^{2}\right)}{n-1} .
$$

Here $\Delta\left(n, X^{2}\right)$ reads

$$
\Delta\left(n, X^{2}\right)=\frac{1}{\rho^{2 n}}-\frac{1}{\left(X^{2}+\rho^{2}\right)^{n}},
$$

with the limit

$$
\lim _{n \rightarrow 0} \frac{\Delta\left(n, X^{2}\right)}{n}=\ln \left(1+\frac{X^{2}}{\rho^{2}}\right)
$$

subsumed, as $X \rightarrow 0, f_{n}$ and $g_{n}$ are all regular. With the above in mind, the explicit solution for $\Phi_{0}$ follows:

$$
\begin{aligned}
\Phi_{0}= & -\frac{c}{\left(X^{2}+\rho^{2}\right)^{\frac{1}{2}}}\left[12 \rho^{2} g_{2}\left(X^{2}, \rho^{2}\right) \bar{\sigma} \cdot \dot{X}\right. \\
& +6 \rho^{2} f_{2}\left(X^{2}, \rho^{2}\right) \bar{\sigma} \cdot X \tau^{a} \chi^{a}+12 f_{2}\left(X^{2}, \rho^{2}\right) \rho \dot{\rho} \bar{\sigma} \cdot X \\
& \left.+\frac{i}{2 \pi^{2} a}\left(f_{2}\left(X^{2}, \rho^{2}\right)+5 \rho^{2} f_{3}\left(X^{2}, \rho^{2}\right)\right) \bar{\sigma} \cdot X\right] \chi,
\end{aligned}
$$

where we have used the zero-mode profile

$$
f=\frac{c}{\left(X^{2}+\rho^{2}\right)^{\frac{3}{2}}},
$$

with $c=\sqrt{2} \rho / \pi$.

In terms of Eq. (A41), the $\Phi_{0}$ contribution to the Lagrangian is

$$
S=\frac{1}{8 m_{H}} \int d^{4} X J_{0}^{\dagger}(X) \Phi_{0}(X) .
$$

Using the fact that $\chi^{a}$ is anti-Hermitian, all the mixing terms vanish, with the exception of

$$
\begin{aligned}
& \frac{6 i}{8 \pi^{2} a m_{H}} \int d^{4} X \frac{c^{2} \rho^{2} X^{2}}{\left(X^{2}+\rho^{2}\right)^{4}} \\
& \quad \times\left(1+\frac{5 \rho^{2}}{X^{2}+\rho^{2}}\right) f_{2}\left(X^{2}, \rho^{2}\right) \chi^{\dagger} \tau^{a} \chi \chi^{a},
\end{aligned}
$$

which couples the spin of the nucleon core and the heavy quarks. After the spatial integration, it reads

$$
\frac{i}{32 m_{H} \pi^{2} a \rho^{2}} \chi^{\dagger} \tau^{a} \chi \chi^{a} .
$$


The diagonal terms give

$$
\begin{aligned}
& -\frac{1}{8 m_{H}}\left[\dot{X}^{2} \chi^{\dagger} \chi \int d^{4} X \frac{12^{2} \rho^{4} c^{2} g_{2}\left(X^{2}, \rho^{2}\right)}{\left(X^{2}+\rho^{2}\right)^{4}}\right. \\
& +\rho^{2} \dot{\rho}^{2} \chi^{\dagger} \chi \int d^{4} X \frac{12^{2} c^{2} X^{2} f_{2}\left(X^{2}, \rho^{2}\right)}{\left(X^{2}+\rho^{2}\right)^{4}} \\
& +\chi^{a \dagger} \chi^{a} \chi^{\dagger} \chi \int d^{4} X \frac{6^{2} c^{2} \rho^{2} X^{2} f_{2}\left(X^{2}, \rho^{2}\right)}{\left(X^{2}+\rho^{2}\right)^{4}} \\
& +\frac{c^{2} \rho^{4}}{4 \pi^{4} a^{2}} \chi^{\dagger} \chi \int d^{4} X \frac{X^{2}\left[f_{2}\left(X^{2}, \rho^{2}\right)+5 \rho^{2} f_{3}\left(X^{2}, \rho^{2}\right)\right]}{\left(X^{2}+\rho^{2}\right)^{4}} \\
& \left.\quad \times\left(1+\frac{5 \rho^{2}}{X^{2}+\rho^{2}}\right)\right]
\end{aligned}
$$

and reduce to

$$
-\chi^{\dagger} \chi\left(\frac{\dot{X}^{2}}{4 m_{H} \rho^{2}}+\frac{1}{4 m_{H}} \frac{\dot{\rho}^{2}}{\rho^{2}}+\frac{1}{4 m_{H}} \dot{a}_{I}^{2}+\frac{25}{6144 m_{H} \pi^{4} a^{2} \rho^{4}}\right)
$$

after integration. Equations (A45)-(A47) yield the final $\Phi_{o}$ contribution to the action

$$
\begin{aligned}
\mathcal{L}_{\Phi_{0}}= & -\chi^{\dagger} \chi\left(\frac{\dot{X}^{2}}{4 m_{H} \rho^{2}}+\frac{1}{4 m_{H}} \frac{\dot{\rho}^{2}}{\rho^{2}}+\frac{1}{4 m_{H}} \dot{a}_{I}^{2}+\frac{25}{6144 m_{H} \pi^{4} a^{2} \rho^{4}}\right) \\
& +\frac{i}{32 m_{H} \pi^{2} a \rho^{2}} \chi^{\dagger} \tau^{a} \chi \chi^{a} .
\end{aligned}
$$

\section{The warping contribution: $\delta L_{\text {warp }}$}

The warping contribution stems from $\tilde{S}_{1}$ and does not have any derivative coupling. More specifically, we have

$$
\begin{aligned}
\delta \tilde{S}_{1}= & \frac{(z+Z)^{2}}{24 m_{H}}\left(\left(3-\frac{2 z^{2}}{z^{2}+x^{2}}\right) f^{\prime 2}+\frac{6 x^{2}+12 z^{2}}{\left(x^{2}+z^{2}+\rho^{2}\right)^{2}} f^{2}\right) \chi^{\dagger} \chi \\
& -\frac{(z+Z)^{2}}{8 m_{H}}\left(\left(1+\frac{2 z^{2}}{x^{2}+z^{2}}\right) f^{\prime 2}+\frac{9 x^{2}+3 z^{2}}{\left(x^{2}+z^{2}+\rho^{2}\right)^{2}} f^{2}\right) \chi^{\dagger} \chi \\
& +\frac{\rho^{2}(z+Z)^{2}}{m_{H}\left(z^{2}+x^{2}+\rho^{2}\right)^{2}} f^{2} \chi^{\dagger} \chi .
\end{aligned}
$$

After spatial integration, Eq. (A49) gives rise to a $\frac{Z^{2}}{\rho^{2}} \chi^{\dagger} \chi$ term as well as a $\chi^{\dagger} \chi$ term, namely

$$
\mathcal{L}_{\text {warp }}=-\frac{37+12 \frac{Z^{2}}{\rho^{2}}}{192 m_{H}} \chi^{\dagger} \chi .
$$

Notice that the $Z^{2}$ contribution is negative, which is consistent with an instability at large $Z$.

\section{The contribution: $\mathcal{L}_{0}$}

To leading order in $\lambda$, this contribution vanishes since $\Phi_{M}$ satisfies the equation of motion. However, there are contributions to $\hat{A}_{M}$ at order $1 / \lambda$,

$\mathcal{L}_{0}=4 a N_{c} \lambda \Phi_{M}^{\dagger} \Phi_{N} \hat{F}_{M N}=8 a N_{c} \lambda \Phi_{M}^{\dagger} \Phi_{N} \partial_{M} \hat{A}_{N}$

To linear order in $\chi^{a}$, we need the explicit solution to $\hat{A}_{M}$,

$$
\hat{A}_{M}=\frac{i}{16 \pi^{2} a \lambda} \frac{\chi^{a} \operatorname{tr} \tau^{a} \sigma_{M N} X_{N}}{2\left(X^{2}+\rho^{2}\right)^{2}} .
$$

With this in mind and using the identities

$$
\sigma_{N M}=i \bar{\eta}_{N M}^{a} \tau^{a}
$$

$$
\bar{\eta}_{N M}^{a} \bar{\eta}_{N M}^{b}=4 \delta^{a b}
$$

we have

$8 a N_{c} \lambda \Phi_{M}^{\dagger} \Phi_{N} \partial_{M} \hat{A}_{N}=\frac{1}{8 m_{H} \pi^{2} a} \frac{\rho^{4} f^{2}}{\left(X^{2}+\rho^{2}\right)^{3}} i \chi^{a} \chi^{\dagger} \tau^{a} \chi$,

which after spatial integration reduces to

$$
\mathcal{L}_{0}=\frac{i}{80 m_{H} \pi^{2} a} \chi^{a} \chi^{\dagger} \tau^{a} \chi
$$

\section{APPENDIX B: COULOMB-BACK REACTION}

Here we provide a complete treatment of the Coulombback interaction contribution. After rescaling $A_{0} \rightarrow i A_{0}$, the Lagrangian for $A^{0}$ reads

$$
\begin{aligned}
\mathcal{L}\left[A_{0}\right]= & \frac{a N_{c}}{2}\left(\vec{\nabla} A_{0}\right)^{2}+\frac{f^{2}}{2 m_{H}} \chi^{\dagger} \chi A_{0}^{2} \\
& +A_{0}\left(\rho^{\mathrm{cl}}+\rho_{0}+\frac{1}{m_{H}} \rho_{1}\right),
\end{aligned}
$$

where $\rho^{\mathrm{cl}}$ is the source without the heavy-quark field

$$
\rho^{\mathrm{cl}}=a N_{c} \nabla^{2} A_{0}^{\mathrm{cl}}=-\frac{3 N_{c}}{\pi^{2}} \frac{\rho^{4}}{\left(x^{2}+\rho^{2}\right)^{4}}
$$

and we have

$$
\begin{aligned}
& \rho_{0}=f^{2} \chi^{\dagger} \chi, \\
& \rho_{1}=\frac{f^{2}}{2} i\left(\chi^{\dagger} \dot{\chi}-\dot{\chi}^{\dagger} \chi\right)+\frac{3}{16 m_{H} \pi^{2} a} \frac{2 \rho^{2}-X^{2}}{\left(X^{2}+\rho^{2}\right)^{2}} f^{2} \chi^{\dagger} \chi .
\end{aligned}
$$

Notice that 


$$
\begin{aligned}
& \frac{3}{16 m_{H} \pi^{2} a} \frac{2 \rho^{2}-X^{2}}{\left(X^{2}+\rho^{2}\right)^{2}} \\
& =\frac{3}{16 m_{H} \pi^{2} a} \frac{f^{2} \rho^{2}}{\left(X^{2}+\rho^{2}\right)^{2}} \chi^{\dagger} \chi \\
& \quad+\frac{3}{64 m_{H} \pi^{2} a} \partial_{N}\left(\frac{x_{N} f^{2}}{\left(x^{2}+\rho^{2}\right)}\right) \chi^{\dagger} \chi
\end{aligned}
$$

originates purely from the Chern-Simons contribution. Given the action for $A_{0}$, at a minimum we have

$$
\begin{aligned}
\mathcal{L}_{\text {Coulumb }}= & -\left(\rho^{\mathrm{cl}}+\rho_{0}+\frac{1}{m_{H}} \rho_{1}\right) \frac{1}{2\left(-a N_{c} \nabla^{2}+\frac{f^{2}}{m_{H}} \chi^{\dagger} \chi\right)} \\
& \times\left(\rho^{\mathrm{cl}}+\rho_{0}+\frac{1}{m_{H}} \rho_{1}\right),
\end{aligned}
$$

which is a complicated function in $\chi^{\dagger} \chi$ and always leads to positive energy. In fact, the $\frac{f^{2}}{m_{H}}$ term in the denominator plays the role of a screening mass which can be seen after certain coordinate transformations.

To estimate how good the first-order expansion is, one can consider the simplest case where the inversion is acting only on the $\rho_{0} \propto f^{2}$. To keep track of the dependence on $\rho$ and $m_{H}$, it is useful to perform the rescaling

$$
X \rightarrow \frac{1}{\sqrt{m}_{y}} \tilde{\rho} \tilde{X}, \quad \rho \rightarrow \frac{1}{\sqrt{m}_{y}} \tilde{\rho} .
$$

As a result we have

$\frac{1}{\left(-a N_{c} \nabla^{2}+\frac{f^{2}}{m_{H}} \chi^{\dagger} \chi\right)} f^{2}=\frac{32}{\tilde{\rho}^{2}} \frac{1}{-\tilde{\nabla}^{2}+\frac{32 \chi^{\dagger} \chi}{m_{H} \tilde{\rho}^{2}} \frac{1}{\left(\tilde{X}^{2}+1\right)^{3}}} \frac{1}{\left(\tilde{X}^{2}+1\right)^{3}}$,

which can be exactly solved as

$\frac{1}{-\tilde{\nabla}^{2}+\frac{32 \chi^{\dagger} \chi}{m_{H} \tilde{\rho}^{2}} \frac{1}{\left(\tilde{X}^{2}+1\right)^{3}}} \frac{1}{\left(\tilde{X}^{2}+1\right)^{3}}=\frac{1}{b}-\frac{\sqrt{\tilde{X}^{2}\left(1+\tilde{X}^{2}\right)} I_{1}\left(\sqrt{\frac{b \tilde{X}^{2}}{1+\tilde{X}^{2}}}\right)}{b I_{1}(\sqrt{b}) \tilde{X}^{2}}$,

with $b=\frac{32 \chi^{\dagger} \chi}{m_{H} \tilde{\rho}}$. Therefore, one has

$$
\begin{aligned}
& f^{2} \frac{1}{\left(-a N_{c} \nabla^{2}+\frac{f^{2}}{m_{H}} \chi^{\dagger} \chi\right)} f^{2} \\
& =\frac{64}{\pi^{2} \tilde{\rho}^{2}} \int d^{4} \tilde{X} \frac{1}{\left(\tilde{X}^{2}+1\right)^{3}}\left(\frac{1}{b}-\frac{\sqrt{\tilde{X}^{2}\left(1+\tilde{X}^{2}\right)} I_{1}\left(\sqrt{\frac{b \tilde{X}^{2}}{1+\tilde{X}^{2}}}\right)}{b I_{1}(\sqrt{b}) \tilde{X}^{2}}\right) .
\end{aligned}
$$

Notice that although the $\frac{1}{b}$ appears to be at variance with power counting, the Taylor expansion

$$
\begin{aligned}
g(b, \tilde{X}) \equiv & \frac{1}{b}-\frac{\sqrt{\tilde{X}^{2}\left(1+\tilde{X}^{2}\right)} I_{1}\left(\sqrt{\frac{b \tilde{X}^{2}}{1+\tilde{X}^{2}}}\right)}{b I_{1}(\sqrt{b}) \tilde{X}^{2}} \\
= & \frac{1}{8\left(\tilde{X}^{2}+1\right)}+\left(-\frac{\tilde{X}^{4}}{192\left(\tilde{X}^{2}+1\right)^{2}}+\frac{\tilde{X}^{2}}{64\left(\tilde{X}^{2}+1\right)}-\frac{1}{96}\right) b \\
& +\left(-\frac{\tilde{X}^{6}}{9216\left(\tilde{X}^{2}+1\right)^{3}}+\frac{\tilde{X}^{4}}{1536\left(\tilde{X}^{2}+1\right)^{2}}\right. \\
& \left.-\frac{\tilde{X}^{2}}{768\left(\tilde{X}^{2}+1\right)}+\frac{7}{9216}\right) b^{2}+\mathcal{O}\left(b^{3}\right)
\end{aligned}
$$

formally converges for any $b$. However, for the case where $\tilde{\rho}=1$ and $\chi^{\dagger} \chi=1$, one has $b=\frac{32}{m_{H}} \approx 8$ for charm and $\approx 3.2$ for bottom, the convergence is poor for the first few terms. To perform an estimate, one can consider the ratio

$$
R(b)=\frac{\int d^{4} \tilde{X} \frac{g(b, \tilde{X})}{(1+\tilde{X})^{3}}}{\int d^{4} \tilde{X} \frac{\lim _{b \rightarrow 0} g(b, \tilde{X})}{\left(1+\tilde{X}^{2}\right)^{3}}}
$$

which is shown in Fig. 2. One can actually show that $R(b)$ is always positive and goes to zero as $b \rightarrow \infty$ or $\tilde{\rho} \rightarrow 0$, which implies a weaker repulsion compared to the leadingorder Coulomb one. However, expanding to leading order in $b$, the potential becomes unbounded from below at large $b$ or small $\rho$. Apparently, this instability is caused by the breakdown of the small $b$ expansion near the core. To fix the instability, we can include the second-order term in the expansion. In fact, in Fig. 2 we note that after including the second-order term, the difference between the full result is

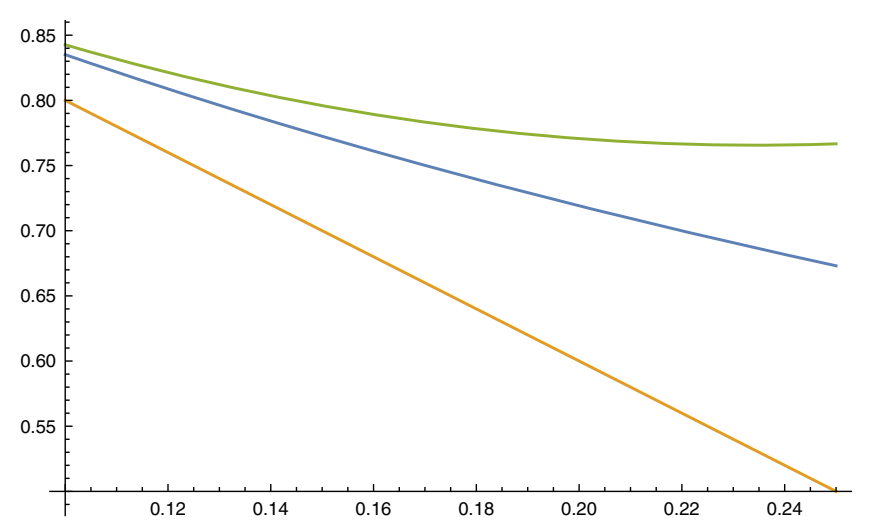

FIG. 2. The ratio $R\left(b \equiv \frac{32}{m_{H}}\right)$ (blue) compared with its first-order (yellow) and second-order (green) Taylor expansion. At $\frac{1}{m_{H}}=\frac{1}{4}$ one has $R(b) \approx 0.67$, while at $\frac{1}{m_{H}}=\frac{1}{10}$ one has $R(b) \approx 0.84$, and as $b \rightarrow \infty, R(b) \rightarrow 0$. At $\frac{1}{m_{H}}=\frac{1}{4}$ the second-order result is about $10 \%$ larger than that of the full result. 
around $10 \%$ for $1 / m_{H} \approx 4$ at $\tilde{\rho} \approx 1$ for the charm quark. It is even better for the bottom quark.

Using the explicit form of the inversion

$$
\begin{aligned}
& \frac{1}{-\tilde{\nabla}^{2}+\frac{b}{\left(\tilde{X}^{2}+1\right)^{3}}}\left(\frac{1}{\left.\tilde{X}^{2}+1\right)^{4}}\right. \\
&=\frac{\tilde{X}^{2}+2}{24\left(X^{2}+1\right)^{2}}-\frac{\left(3 \tilde{X}^{4}+9 \tilde{X}^{2}+7\right)}{1152\left(\tilde{X}^{2}+1\right)^{3}} b+\frac{\left(16 \tilde{X}^{6}+64 \tilde{X}^{4}+86 \tilde{X}^{2}+39\right)}{92160\left(\tilde{X}^{2}+1\right)^{4}} b^{2} \\
&-\frac{\left(130 \tilde{X}^{8}+650 \tilde{X}^{6}+1220 \tilde{X}^{4}+1020 \tilde{X}^{2}+321\right)}{11059200\left(\tilde{X}^{2}+1\right)^{5}} b^{3} \\
&+\frac{\left(1485 \tilde{X}^{10}+8910 \tilde{X}^{8}+21365 \tilde{X}^{6}+25605 \tilde{X}^{4}+15345 \tilde{X}^{2}+3681\right)}{1857945600\left(\tilde{X}^{2}+1\right)^{6}} b^{4}
\end{aligned}
$$

and

$$
\begin{aligned}
& \frac{1}{-\tilde{\nabla}^{2}+\frac{b}{\left(\tilde{X}^{2}+1\right)^{3}}} \frac{1}{\left(\tilde{X}^{2}+1\right)^{5}} \\
= & \frac{\tilde{X}^{4}+3 \tilde{X}^{2}+3}{48\left(\tilde{X}^{2}+1\right)^{3}}-\frac{\left(18 \tilde{X}^{6}+72 \tilde{X}^{4}+98 \tilde{X}^{2}+47\right)}{11520\left(\tilde{X}^{2}+1\right)^{4}} b+\frac{\left(50 \tilde{X}^{8}+250 \tilde{X}^{6}+470 \tilde{X}^{4}+395 \tilde{X}^{2}+126\right)}{460800\left(\tilde{X}^{2}+1\right)^{5}} b^{2} \\
& -\frac{\left(575 \tilde{X}^{10}+3450 \tilde{X}^{8}+8275 \tilde{X}^{6}+9925 \tilde{X}^{4}+5958 \tilde{X}^{2}+1434\right)}{77414400\left(\tilde{X}^{2}+1\right)^{6}} b^{3} \\
& +\frac{\left(26355 \tilde{X}^{12}+184485 \tilde{X}^{10}+537355 \tilde{X}^{8}+833875 \tilde{X}^{6}+727335 \tilde{X}^{4}+338205 \tilde{X}^{2}+65523\right)}{52022476800\left(\tilde{X}^{2}+1\right)^{7}} b^{4} .
\end{aligned}
$$

Equation (27) follows to order $\mathcal{O}\left(1 / m_{H}^{2}\right)$.

\section{APPENDIX C: DETAILS OF THE HEAVY PENTAQUARK MASSES}

Here we detail the various contributions to the mass spectra recorded in Tables I and II. For completeness, we recall that we fix $M_{D}=1.87 \mathrm{GeV}$ to reproduce the D-meson mass in Eq. (7) and fix $M_{K K}=0.475 \mathrm{GeV}$ to reproduce the $M_{\Lambda_{c}}=2.286 \mathrm{GeV}$. As result, we have for the charmed heavy-light hadrons recorded in Table I

$$
\begin{aligned}
& M_{\Lambda_{c}}=m_{H}+m_{N}-0.82 M_{K K}+0.253 \frac{M_{K K}^{2}}{m_{H}}=2.286 \mathrm{GeV} \\
& M_{\Sigma_{c}}\left(\frac{1}{2}\right)=m_{H}+m_{N}-0.234 M_{K K}+0.203 \frac{M_{K K}^{2}}{m_{H}}=2.557 \mathrm{GeV} \\
& M_{\Sigma_{c}}\left(\frac{3}{2}\right)=m_{H}+m_{N}-0.154 M_{K K}+0.203 \frac{M_{K K}^{2}}{m_{H}}=2.596 \mathrm{GeV}, \\
& M_{\Lambda_{c}^{\star}}(P=-1)=m_{H}+m_{N}-0.82 M_{K K}+\frac{2}{\sqrt{6}} M_{K K}+0.321 \frac{M_{K K}^{2}}{m_{H}}=2.683 \mathrm{GeV}, \\
& M_{\Lambda_{c}^{\star}}(P=1)=m_{H}+m_{N}+0.107 M_{K K}+0.253 \frac{M_{K K}^{2}}{m_{H}}=2.726 \mathrm{GeV}, \\
& P_{c}\left(J=\frac{1}{2}, S=0\right)=2 m_{H}+m_{N}-0.078 M_{K K}+0.404 \frac{M_{K K}^{2}}{m_{H}}=4.360 \mathrm{GeV},
\end{aligned}
$$




$$
\begin{gathered}
P_{c}\left(J=\frac{1}{2}, S=1\right)=2 m_{H}+m_{N}-0.119 M_{K K}+0.404 \frac{M_{K K}^{2}}{m_{H}}=4.341 \mathrm{GeV}, \\
P_{c}\left(J=\frac{3}{2}, S=1\right)=2 m_{H}+m_{N}-0.05 M_{K K}+0.404 \frac{M_{K K}^{2}}{m_{H}}=4.373 \mathrm{GeV}
\end{gathered}
$$

For the bottom heavy-light hadrons we fix the heavy-light meson mass $m_{H}=(5.28-0.168) \mathrm{GeV}=10.76 M_{K K}$. The bottom heavy-light mass spectra recorded in Table II follow from

$$
\begin{aligned}
& M_{\Lambda_{b}}=m_{H}+m_{N}-0.958 M_{K K}+0.253 \frac{M_{K K}^{2}}{m_{H}}=5.608 \mathrm{GeV} \\
& M_{\Sigma_{b}}\left(\frac{1}{2}\right)=m_{H}+m_{N}-0.207 M_{K K}+0.203 \frac{M_{K K}^{2}}{m_{H}}=5.962 \mathrm{GeV} \\
& M_{\Sigma_{b}}\left(\frac{3}{2}\right)=m_{H}+m_{N}-0.174 M_{K K}+0.203 \frac{M_{K K}^{2}}{m_{H}}=5.978 \mathrm{GeV} \\
& M_{\Lambda_{b}^{*}}(P=-1)=m_{H}+m_{N}-0.958 M_{K K}+\frac{2}{\sqrt{6}} M_{K K}+0.321 \frac{M_{K K}^{2}}{m_{H}}=5.998 \mathrm{GeV} \\
& M_{\Lambda_{b}}^{\star}(P=1)=m_{H}+m_{N}-0.072 M_{K K}+0.253 \frac{M_{K K}^{2}}{m_{H}}=6.029 \mathrm{GeV} \\
& P_{b}\left(J=\frac{1}{2}, S=0\right)=2 m_{H}+m_{N}-0.0393 M_{K K}+0.404 \frac{M_{K K}^{2}}{m_{H}}=11.163 \mathrm{GeV} \\
& P_{b}\left(J=\frac{1}{2}, S=1\right)=2 m_{H}+m_{N}-0.056 M_{K K}+0.404 \frac{M_{K K}^{2}}{m_{H}}=11.155 \mathrm{GeV} \\
& P_{b}\left(J=\frac{3}{2}, S=1\right)=2 m_{H}+m_{N}-0.030 M_{K K}+0.404 \frac{M_{K K}^{2}}{m_{H}}=11.196 \mathrm{GeV}
\end{aligned}
$$

[1] R. Aaij et al. (LHCb Collaboration), Observation of a Narrow Pentaquark State, $P_{c}(4312)^{+}$, and of Two-Peak Structure of the $P_{c}(4450)^{+}$, Phys. Rev. Lett. 122, 222001 (2019).

[2] R. Aaij et al. (LHCb Collaboration), Observation of $J / \psi p$ Resonances Consistent with Pentaquark States in $\Lambda_{b}^{0} \rightarrow J / \psi K^{-} p$ Decays, Phys. Rev. Lett. 115, 072001 (2015).

[3] Y. Liu and I. Zahed, Heavy baryons and their exotics from instantons in holographic QCD, Phys. Rev. D 95, 116012 (2017).

[4] J.-J. Wu, R. Molina, E. Oset, and B. S. Zou, Prediction of Narrow $N^{*}$ and $\Lambda^{*}$ Resonances with Hidden Charm above 4 GeV, Phys. Rev. Lett. 105, 232001 (2010).
[5] M. Karliner and J. L. Rosner, New Exotic Meson and Baryon Resonances from Doubly Heavy Hadronic Molecules, Phys. Rev. Lett. 115, 122001 (2015).

[6] T. J. Burns, Phenomenology of $P_{c}(4380)^{+}, P_{c}(4450)^{+}$and related states, Eur. Phys. J. A 51, 152 (2015).

[7] J.-M. Richard, Exotic hadrons: Review and perspectives, Few-Body Syst. 57, 1185 (2016).

[8] R. F. Lebed, R. E. Mitchell, and E. S. Swanson, Heavyquark QCD exotica, Prog. Part. Nucl. Phys. 93, 143 (2017).

[9] A. Esposito, A. Pilloni, and A. D. Polosa, Multiquark resonances, Phys. Rep. 668, 1 (2017).

[10] S. L. Olsen, T. Skwarnicki, and D. Zieminska, Nonstandard heavy mesons and baryons: Experimental evidence, Rev. Mod. Phys. 90, 015003 (2018). 
[11] F.-K. Guo, C. Hanhart, U.-G. Meißner, Q. Wang, Q. Zhao, and B.-S. Zou, Hadronic molecules, Rev. Mod. Phys. 90, 015004 (2018).

[12] M. Karliner, J. L. Rosner, and T. Skwarnicki, Multiquark states, Annu. Rev. Nucl. Part. Sci. 68, 17 (2018).

[13] L. Maiani, A. D. Polosa, and V. Riquer, The new pentaquarks in the diquark model, Phys. Lett. B 749, 289 (2015).

[14] R. F. Lebed, The pentaquark candidates in the dynamical diquark picture, Phys. Lett. B 749, 454 (2015).

[15] M. I. Eides, V. Yu. Petrov, and M. V. Polyakov, New LHCb pentaquarks as hadrocharmonium states, Mod. Phys. Lett. A 35, 2050151 (2020).

[16] M.-L. Du, V. Baru, F.-K. Guo, C. Hanhart, U.-G. Meißner, J. A. Oller, and Q. Wang, Revisiting the nature of the $P_{c}$ pentaquarks, J. High Energy Phys. 08 (2021) 157.

[17] E. V. Shuryak, Hadrons containing a heavy quark and QCD sum rules, Nucl. Phys. B198, 83 (1982).

[18] N. Isgur and M. B. Wise, Spectroscopy with Heavy Quark Symmetry, Phys. Rev. Lett. 66, 1130 (1991).

[19] M. A. Nowak, M. Rho, and I. Zahed, Chiral effective action with heavy quark symmetry, Phys. Rev. D 48, 4370 (1993).

[20] W. A. Bardeen and C. T. Hill, Chiral dynamics and heavy quark symmetry in a solvable toy field theoretic model, Phys. Rev. D 49, 409 (1994).

[21] M. A. Nowak, M. Praszalowicz, M. Sadzikowski, and J. Wasiluk, Chiral doublers of heavy light baryons, Phys. Rev. D 70, 031503 (2004).

[22] B. Aubert et al. (BABAR Collaboration), Observation of a Narrow Meson Decaying to $D_{s}^{+} \pi^{0}$ at a Mass of $2.32 \mathrm{GeV} / c^{2}$, Phys. Rev. Lett. 90, 242001 (2003).

[23] D. Besson et al. (CLEO Collaboration), Observation of a narrow resonance of mass $2.46 \mathrm{GeV} / c^{2}$ decaying to $D_{s}^{*+} \pi^{0}$ and confirmation of the $D_{s J}^{*}(2317)$ state, Phys. Rev. D 68 , 032002 (2003); 75, 119908(E) (2007).

[24] J. M. Maldacena, The large $N$ limit of superconformal field theories and supergravity, Int. J. Theor. Phys. 38, 1113 (1999).

[25] J. Erlich, E. Katz, . T. Son, and M. A. Stephanov, QCD and a Holographic Model of Hadrons, Phys. Rev. Lett. 95, 261602 (2005).

[26] T. Sakai and S. Sugimoto, Low energy hadron physics in holographic QCD, Prog. Theor. Phys. 113, 843 (2005).

[27] T. Fujiwara, T. Kugo, H. Terao, S. Uehara, and K. Yamawaki, Nonabelian anomaly and vector mesons as dynamical gauge bosons of hidden local symmetries, Prog. Theor. Phys. 73, 926 (1985).

[28] Y. Liu and I. Zahed, Holographic heavy-light chiral effective action, Phys. Rev. D 95, 056022 (2017).

[29] Y. Liu and I. Zahed, Heavy and strange holographic baryons, Phys. Rev. D 96, 056027 (2017).
[30] S.-W. Li, Holographic heavy-baryons in the WittenSakai-Sugimoto model with the D0-D4 background, Phys. Rev. D 96, 106018 (2017).

[31] D. Fujii and A. Hosaka, Heavy baryons in holographic QCD with higher dimensional degrees of freedom, Phys. Rev. D 101, 126008 (2020).

[32] H. Hata, T. Sakai, S. Sugimoto, and S. Yamato, Baryons from instantons in holographic QCD, Prog. Theor. Phys. 117, 1157 (2007).

[33] K. Hashimoto, T. Sakai, and S. Sugimoto, Holographic baryons: Static properties and form factors from gauge/ string duality, Prog. Theor. Phys. 120, 1093 (2008).

[34] K.-Y. Kim and I. Zahed, Electromagnetic baryon form factors from holographic QCD, J. High Energy Phys. 09 (2008) 007.

[35] H. Hata and M. Murata, Baryons and the Chern-Simons term in holographic QCD with three flavors, Prog. Theor. Phys. 119, 461 (2008).

[36] K. Hashimoto, N. Iizuka, T. Ishii, and D. Kadoh, Three-flavor quark mass dependence of baryon spectra in holographic QCD, Phys. Lett. B 691, 65 (2010).

[37] P. H. C. Lau and S. Sugimoto, Chern-Simons five-form and holographic baryons, Phys. Rev. D 95, 126007 (2017).

[38] I. Zahed and G. E. Brown, The Skyrme model, Phys. Rep. 142, 1 (1986).

[39] M. Rho, D. O. Riska, and N. N. Scoccola, The energy levels of the heavy flavor baryons in the topological soliton model, Z. Phys. A 341, 343 (1992).

[40] Y.-H. Lin and B.-S. Zou, Strong decays of the latest LHCb pentaquark candidates in hadronic molecule pictures, Phys. Rev. D 100, 056005 (2019).

[41] H. G. Dosch, G. F. de Teramond, and S. J. Brodsky, Supersymmetry across the light and heavy-light hadronic spectrum, Phys. Rev. D 92, 074010 (2015).

[42] J. Sonnenschein and D. Weissman, Excited mesons, baryons, glueballs and tetraquarks: Predictions of the holography inspired stringy hadron model, Eur. Phys. J. C 79, 326 (2019).

[43] R. Aaij et al. (LHCb Collaboration), Evidence for a new structure in the $J / \psi p$ and $J / \psi \bar{p}$ systems in $B_{s}^{0} \rightarrow J / \psi p \bar{p}$ decays, arXiv:2108.04720.

[44] M. Rho and I. Zahed, The Multifaceted Skyrmion, 2nd ed. (World Scientific, Singapore, 2016), https://www .worldscientific.com/doi/pdf/10.1142/9710.

[45] Y. Liu, M. A. Nowak, and I. Zahed, following paper, Holographic charm and bottom pentaquarks. II. Open and hidden decay widths, Phys. Rev. D 104, 114022 (2021).

[46] Y. Liu, K. A. Mamoy, M. A. Nowak, and I. Zahedx, this issue, Holographic charm and bottom pentaquarks. III. Excitations through photo-production of heavy mesons, Phys. Rev. D 104, 114023 (2021). 